\title{
On a class of mean field solutions of the Monge problem for perfect and self-interacting systems
}

\author{
Philippe Choquard*
}

August 18, 2010

\begin{abstract}
The Monge problem [23], [27], as reformulated by Kantorovich [19], [20] is that of the transportation, at a minimum "cost", of a given mass distribution from an initial to a final position during a given time interval. It is an optimal transport problem [28, sects. 1,2]. Following the fluid mechanical solution provided by Benamou and Brenier for quadratic cost functions [4] ,[28, sects. 5.4, 8.1] and, by analogy with the fixed end problem in Analytical Mechanics, Lagrangian formulations are needed to solve this boundary value problem in time. They are also needed to determine the Actions as time integral of Lagrangians, that are measures of the "cost"of the transportations [4, proposition 1.1]. Four versions of explicit constructions of Lagrangians are proposed in section 3. They are associated to the Hamiltonians of perfect and self-interacting systems presented in section 2. These Hamiltonians are expressed in function of pairs of the well known canonically conjugated Clebsch variables, namely mass densities and velocity potentials [14], [15]. The first version consists in the elimination of the velocity potentials as a function of the densities and their time derivatives by inversion of the continuity equations derived from given Hamiltonians. The second version consists in the elimination of the gradient of the velocity potentials from the continuity equations thanks to the introduction of vector valued applications such that their divergences give the mass densities. It turns out that, up to a sign factor, these vector fields are canonically conjugated to Euler velocity fields. The third version is a generalization in $n \mathrm{D}$ of Gelfand mass coordinate, a constant of the motion in $1 \mathrm{D}$ [17], by the introduction of n-dimensional vector valued applications
\end{abstract}

*EPFL SB-DO, MA A1 354 (Bâtiment MA), Station 8, CH-1015 Lausanne, Switzerland. E-Mail address: philippe.choquard@epfl.ch 
that enable to determine the mass densities as the determinant of their Jacobian matrices. Comparison of this set of mass coordinates with other sets of constants of the motion familiar in Fluid Dynamics is made in sub-section 3.3. Note that version two and three are identical for one-dimensional problems. The fourth version is based on the introduction of the Lagrangian coordinates that describe the characteristics of the different models and are parametrized by the former auxiliary vector fields. As illustrations, weak solutions of several models of Coulombian and Newtonian systems known in Plasma Physics and in Cosmology, respectively, with spherically symmetric boundary densities are given in section 4 . However, and up to one exception given in the sub-section 3.3 , calculations of the actions associated to these illustrations are not reported in this paper, nor the important analysis of the convexity-concavity properties of our Lagrangians. Lastly, and for the same models as those evoked above, a survey of past work concerning weak solutions of the Cauchy problem obeying the Hopf-Lax variational principle extended to negative time and having correlated initial conditions is given in the Introduction as well as the derivation of the continuum fluid limit from many particle Hamiltonians.

Keyword: Perfect, Coulombian and Newtonian fluids; Boundary value problems in time; explicit Lagrangian formulations; Weak Mean Field solutions..

PACS (2010): 03.50.-z; 02.60.Lj; 02.60.Nm; 04.20.Fy; 04.40.-b.

$M S C$ (2010): 35Q35; 35Q70; 34K10; 35D30; 34L30.

\section{Contents}

1 Introduction $\quad 3$

2 The models, their Hamiltonians and equations of motion 6

3 Lagrangian formulations of the Monge problem 9

3.1 First version . . . . . . . . . . . . . . . . . . . . . 10

3.2 Second version . . . . . . . . . . . . . . . . . . . . 14

3.3 Third version . . . . . . . . . . . . . . . . . . . . 15

3.4 Fourth version . . . . . . . . . . . . . . 18

4 Examples of integrable solutions of (3.52) 19

4.1 The $P F^{1}$ model . . . . . . . . . . . . . . . . . . . . . . . . . . . . . . 21

4.2 The $C_{1}^{1}$ and $N_{1}^{1}$ models . . . . . . . . . . . . . . . . 23

4.3 The $C_{2}^{1}$ model . . . . . . . . . . . . . . . . 26 


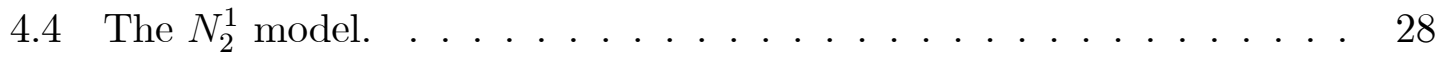

4.5 The $P F^{n}$ model for $n>1 \ldots \ldots \ldots \ldots$

5 Appendices 32

5.1 PDE for $\mathbf{W}(\mathbf{x}, \mathbf{y}, \rho)$ with $1<n \leq 3 \ldots \ldots \ldots \ldots$

5.2 Continuity equation for $\rho(\mathrm{x}, t) \ldots \ldots \ldots \ldots \ldots \ldots \ldots \ldots$

5.3 Explicit $\boldsymbol{\xi}$ of $\mathbf{x}$ relations for radially symmetric boundary densities . . 34

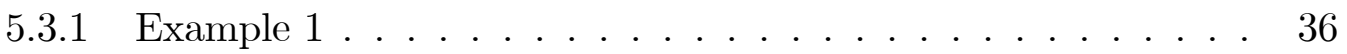

$5.3 .2 \quad$ Example $2 \ldots \ldots \ldots \ldots \ldots \ldots$

5.3 .3 Example $3 \ldots \ldots \ldots \ldots \ldots \ldots$

$\begin{array}{ll}\text { References } & 37\end{array}$

\section{Introduction}

In an earlier paper [8], a non-local Hamiltonian classical field theory of inviscid, compressible and vorticity-free liquids, initially modeled by many-particle Hamiltonians [8, eqn. 3], was set up, using pairs of collective and canonically conjugate variables, to describe the strongly correlated motion of their constituents subject to pair interactions of short and/or long range. For simple systems such as assemblies of identical and massive particles, the natural collective variables are known to be the particle or the mass densities. Advantages of these variables are their invariance with respect to the permutation group of the particles and the fact that the potential energy resulting from pair interactions among the particles is quadratic in the densities, up to self-energy terms.

Considering next the conjugate variables, it was shown that, as a result of the identity required between the Actions expressed as a function of the cartesian coordinates of the models (at two different times) and that expressed as a function of the corresponding particle or mass densities (the collective variables at two different times), and of the ensuing equations relating their derivatives with respect to the corresponding coordinates (cartesian and collective), [8, eqn. 18, sects. 1 \& 6] they are given by the momentum or by the velocity potentials, respectively. For finite systems, the two pairs of canonically conjugate variables are equivalent, as illustrated by the Hamiltonians given in [8, eqns. $29 \& 32]$. For infinite systems, this is no longer true [8, sect. 4]; particle densities come into play in the thermodynamic limit of Statistical Mechanics and mass densities in the continuum limit of Fluid Dynamics. Together with the velocity potentials, they constitute the well known pairs of canonically conjugate Clebsch variables It is this case which applies in the present 
paper and the Hamiltonian of a self-gravitating fluid in 3D ( $\mathrm{D}$ for dimension), is given as example in [8, eqn. 42]. It is notably shown why and how the self-energy terms vanish in that limit.

Similarly, the continuum limit of several many-particle Hamiltonians can be performed and gives rise to Hamiltonians depending upon the corresponding velocity potentials and mass densities. A few representative examples are given in section 2 . The equations of motion resulting from these Hamiltonians are the continuity equations for the densities, the Bernoulli equations for the velocity potentials and the Euler equations for the velocity fields, all generally non-local and non-linear. Their solutions with prescribed initial conditions represent the motions of test or tagged particles that experience the forces due to all the real particles or constituents of the models but do not react on them. The resulting trajectories, called characteristics, are the Mean Field solutions of the problems at hand. These solutions, called weak or generalized, can be both regular and singular and they have to obey Hopf-Lax' variational principle [28, sect. 5.4.6] or formula as shown in [10, eqn. 28], [11, eqn. 22], [12, eqn. 44] and [13, eqn. 13]. This latter formula is the fluid mechanical equivalent of the "equal area rule" or Maxwell construction or convex envelope construction of Free Energies in Statistical Mechanics .

In the next paper [9], an instructive comparison has been made between the equations that govern the equilibrium properties of classical ferro- and antiferromagnets in the Mean Field Approximation and those that are relevant for the space-time evolution of a compressible Burgers' fluid in 1D. A consequence of this analogy is the necessity to investigate weak solutions of the Burgers equation for negative time corresponding to the Curie-Weiss transition in ferromagnets and, thus, implying an extension of the Hopf-Lax formula to negative time [9, section 3], Self-explanatory illustrations of the space-time evolution of the density and velocity field for positive and negative times are shown in [9, Figs. 1 \& 2, p. 851].

In subsequent papers [10], [11] and [13], theoretical and numerical investigations concerning a class of integrable models in 1,2 and 3D consisting of Coulombian systems known in Plasma Physics and of Newtonian systems known in Cosmology were reported. Their Hamiltonians are particular cases of a generic one [13, eqn.1] and the integrability of the corresponding equations of motion results from the following facts. Firstly, their interaction potential being Coulombian or Newtonian, local PDE's in the densities are obtained after taking the laplacian or the divergence of the Bernoulli or Euler equations, respectively; secondly, and assuming radially symmetric densities, re-integration of the radial divergences results in Gelfand type of mass coordinates which are constants of the motion [11, eqn. 19], [13, eqn. 12] and (5.18). Another important feature concerns the initial conditions satisfied by 
the mass densities and by the velocity fields. They are said to be correlated in the sense that the initial densities are proportional to the determinant of the Hessian matrix of the initial velocity potential [11, cf. footnote p. 173]. Lastly, they satisfy the Hopf-Lax variational principle extended to negative time [9, sect. 3]. A remarkable and unique consequence of the preceding conditions and properties is that for all the one-dimensional models treated in [10] and [11], the weak solutions of the Cauchy problems are of the Burgers type, the time variable being replaced by model dependant functions of the time [10, eqn. 18, Figs. 5, 6, 9] and [11, eqns. $31 \& 34]$.

In this paper we develop a classical field theoretical formulation, extended to self-interacting systems, of the celebrated Monge mass transfer problem [23], [27], defined above and since it is a boundary value problem in time, then, by analogy with the fixed-end problem in Analytical Mechanics, the relevant functionals are Lagrangians which depend upon the densities or other generalized coordinates and their time derivative and which are obtained from given Hamiltonians by suitable Legendre transforms.

Section 2 is devoted to the presentation of the generic Hamiltonian quoted above and of the resulting equations of motion, namely the continuity, the non-local Bernoulli, the ensuing Euler and div-Euler equations from which all the particular models can be specified.

Owing to the importance of the subject, four Lagrangian formulations of the Monge problem are developed in the third section. By analogy with the elimination of the momenta as functions of the coordinates and their time derivatives in Analytical Mechanics, the first one consists in the elimination of the velocity potentials as functions of the mass densities and their time derivatives by inversion of the continuity equations. By means of natural Legendre transforms we obtain the first Lagrangians, labeled $L_{1}\left(\partial_{t} \rho, \rho\right)(3.10)$ useful for further theoretical work, with the kinetic energies expressed as a quadratic form in the time derivatives of the mass densities convoluted with the density dependent inversion kernels and the potential energies, as a quadratic form in the mass densities convoluted with the interaction potentials. The second ones consists in the elimination of the gradient of the velocity potentials from the continuity equations thanks to the introduction of vector valued applications $\boldsymbol{\eta}(\mathbf{x}, \mathbf{t})$ such that their divergences give the mass densities $\rho(\mathbf{x}, t)$. The corresponding Lagrangians are labeled $L_{2}\left(\partial_{t} \boldsymbol{\eta},\langle\boldsymbol{\nabla}, \boldsymbol{\eta}\rangle\right)$ (3.28). Their kinetic energies become local functions of the space and time derivatives of these field variables and it turns out that, up to a sign factor, these vector fields are canonically conjugated to Euler velocity fields. This means that strict canonicity can be obtained in permuting the roles of generalized coordinate and conjugate momentum. The third version 
is based on the introduction of auxiliary vector valued applications $\boldsymbol{\xi}(\mathbf{x}, t)$ that are n-dimensional generalizations of Gelfand mass coordinates in $1 D$ in the sense that the $n$ components of $\boldsymbol{\xi}(\mathbf{x}, t)$ are constants of the motion, like in [26, eqn. 3.4]. An important, although not unique, advantage of our mass coordinates is that the mass densities are given by the determinant of their Jacobian matrix and we obtain the third Lagrangians, labeled $L_{3}\left(\partial_{t} \boldsymbol{\xi}\right.$, det $\left.\left(D_{\mathbf{x}} \boldsymbol{\xi}\right)\right)$ (3.36), which are functionals of both time and spatial derivatives of $\boldsymbol{\xi}(\mathbf{x}, t)$. The last and very useful version is based on the Lagrangian coordinates $\mathbf{x}(\boldsymbol{\xi}, t)$ of the characteristics of the models parametrized by $\boldsymbol{\xi}$. We obtain the fourth Lagrangians, labeled $L_{4}\left(\partial_{t} \mathbf{x}, \mathbf{x}\right)(3.51)$, which are functionals of $\mathbf{x}(\boldsymbol{\xi}, t)$ and their time derivatives and we establish the generic equations of motion (3.52).

Section 4 is dedicated to the presentation of weak solutions of (3.52) and (4.4) for several models obeying linear second order ODE's with or without time-dependant coefficients. Their integrability, like that of all the models dealt with in this paper, relies on three properties: i) separability of the motion of the barycenter, $\mathbf{b}(t)$, of the mass densities from the motion, $\mathbf{y}(\boldsymbol{\xi}, t)$, of their constituents relative to this center (what could be called the "barycenter theorem"), a property expressed through the equation (4.1), ii) parallelism of $\mathbf{y}(\boldsymbol{\xi}, t)$ with $\boldsymbol{\xi}$ for all times with the purpose of minimizing the "cost" of the mass transportation and iii) spherically symmetric boundary densities that entail that if $\mathbf{y}(\boldsymbol{\xi}, t)$ is a solution then $-\mathbf{y}(\boldsymbol{\xi}, t)$ is also a solution. The corresponding trajectories, called sister-trajectories, may or may not collide and if they do, they generate finite, semi-infinite or even periodic shocklines, depending upon the force fields that they are subject to. A particular attention is paid in this section to the identification, in their parameter space, of the regularity and singularity domains of the weak solutions of the models investigated.

\section{The models, their Hamiltonians and equations of motion}

We consider the models dealt with in references [9], [10], [11] and [13], i.e., the inviscid, compressible, vorticity-free, perfect and self-interacting one component systems subject to Coulombian and Newtonian interactions in $n \mathrm{D}$, with $n=1,2,3$, including those with densities departing from uniform backgrounds and those implying universes in expansion.

Let $\rho(\mathbf{x}, t)$, the mass density and $S(\mathbf{x}, t)$, the velocity potential be the canonically conjugate Clebsch variables for all models. We have that $(\mathbf{x}, t) \in \mathbb{R}^{n} \times \mathbb{R}, \rho \in \mathbb{R}^{+}$, $S \in \mathbb{R}$, and assume that $\rho$ is Borel measurable with total mass $M$ and that $S$ 
is globally Lipschitz continuous. The force fields being the gradients of potential functions are curl-free and so are the acceleration and velocity fields. For the latter, we set $\mathbf{U}(\mathbf{x}, t)=\boldsymbol{\nabla} S(\mathbf{x}, t)$, the symbol $\boldsymbol{\nabla}$ meaning the gradient operator. Like in [13, eqn.1], the generic Hamiltonian expressed in terms of the comoving coordinates $\mathbf{x}$ (in the cases of the cosmological models) is given, in the continuum and in a dimensionality- dependent limit, by

$$
\begin{aligned}
H(S, \rho)= & T(S, \rho)+V\left(\rho-\rho_{b}\right)= \\
& \frac{1}{2 a(t)^{2}} \int d \mathbf{x} \rho(\mathbf{x}, t)(\nabla S(\mathbf{x}, t))^{2} \\
& +\frac{g}{2 a(t)} \iint d \mathbf{x} d \mathbf{y}\left(\rho(\mathbf{x}, t) v-\rho_{b}\right) \psi(|\mathbf{x}-\mathbf{y}|)\left(\rho(\mathbf{y}, t)-\rho_{b}\right)
\end{aligned}
$$

where all the integrations are carried over $\mathbb{R}^{n}$, in the sequel as well, unless otherwise specified, and where $a(t)$ is the dimensionless cosmological scale factor discussed below. We recall that the total mass $M=N m$, is prescribed in the continuum limit with the number of particles $N \rightarrow \infty$ whereas their mass $m \rightarrow 0$. We recall also that, in $1 \mathrm{D}$, we have the motion, normal to their plane, of stratified sheets of matter of given surface number density $\kappa^{2}$ with $g=g_{a}(1)=-4 \pi G \kappa^{2}$, (a, for attractive) in the Newtonian cases where $G$ is the gravitational constant and $g=g_{r}(1)=4 \pi(e / m)^{2} \kappa^{2}$ $(r$,for repulsive) in the Coulombian cases, where the ratio $e / m=\widetilde{\gamma}$ (charge of the particle divided by their mass) in the continuum limit, while $e \rightarrow 0$ and $m \rightarrow 0$. Moreover, the one-dimensional prescribed background density is $\rho_{b}(1)=m \nu$, with $m \rightarrow 0$ and the concentration of the sheets $\nu \rightarrow \infty$. Lastly, with $x=|\mathbf{x}|$, the interaction potential is $\psi(x, 1)=-x / 2$. In $2 \mathrm{D}$, we have the motion of parallel lines of matter, normal to the plane, of given linear density $\kappa$, with $g=g_{a}(2)=-4 \pi G \kappa$, and $g=g_{r}(2)=4 \pi(e / m)^{2} \kappa, \rho_{b}(2)=m \nu^{2}, \nu^{2} \rightarrow \infty$ being the concentration of the lines in the plane and $\psi(x, 2)=-(1 / 2 \pi) \log x$. In 3D, we have the motion of point particles with $g=g_{a}(3)=-4 \pi G, g_{r}(3)=4 \pi(e / m)^{2}, \rho_{b}(3)=m \nu^{3}, \nu^{3} \rightarrow \infty$ being the number density of the particles and $\psi(x, 3)=1 /(4 \pi x)$. We observe that the Jeans (imaginary) frequencies and the plasma frequencies come into play in the form: $g_{a}(n) \rho_{b}(n)=\Omega_{J}(n)^{2}$ and $g_{r}(n) \rho_{b}(n)=\Omega_{P}(n)^{2}$.

The different models can now be characterized. In the perfect fluids cases, labeled $P F^{n}$, we have $a(t)=1$ and $g=0$. In the Coulombian cases, we consider first the purely repulsive ones, labeled $C_{1}^{n}$, with $a(t)=1, \rho_{b}(n)=0$, and $g=g_{r}(n)$, and then, those with densities departing from a homogenous and neutralizing background, called One-Component-Plasma (OCP), labeled $C_{2}^{n}$ with $a(t)=1, g=g_{r}(n)$ and $\rho_{b}(n)>0$. In the Newtonian cases, we consider first the purely attractive one, 
labeled $N_{1}^{n}$, with $g=-g_{a}(n), a(t)=1$ and $\rho_{b}(n)=0$, and two cosmological models. The latter consist of pressure-less (cold), collision-less (dark) dust (matter) in a flat expanding universe characterized by cosmic scale factors depending or not upon the cosmological constant $\Lambda$, labeled $N_{2}^{n}$ and $N_{3}^{n}$, respectively. In the $N_{2}^{n}$ cases, $a(t)=\left(t / \tilde{t}_{0}\right)^{2 / 3}$, with $\tilde{t}_{0}^{-2}=\frac{3}{2} \Omega_{J}(n)^{2}, g=-g_{a}(n)$ and $\rho_{b}(n)>0$, we have the so called Standard Cold Dark Matter models (SCDM) [24, p.81]. In the $N_{3}^{n}$ cases, called $\Lambda$ CDM models [16, p.248] we have $a(t)=\left(\sinh \left(\lambda t / \tilde{t}_{0}\right) / \lambda\right)^{2 / 3}$ where $\lambda^{2}$ is the ratio of the vacuum energy to the mass energy, i.e., in $3 \mathrm{D}, \Lambda c^{2} / 8 \pi G \rho_{b} \cong 0.7 / 0.3[16$, p 254]. We recall that one of the purposes of the cosmological models is to understand the formation of large structures of the universe [7], [22].

As mentioned in the Introduction, results of theoretical and numerical investigations concerning solutions of the Cauchy problem for the model $P F^{1}$ are reported in [9],for the model $C_{1}^{1}$ and $N_{1}^{1}$, in [10],for the models $C_{2}^{1}, N_{2}^{1}$ and $N_{3}^{1}$, in [11] and for the model $N_{3}^{2}$, in [13].

We proceed with Hamilton equations of motion which are $\partial_{t} \rho=\delta H / \delta S$ and $\partial_{t} S=$ $-\delta H / \delta \rho$, the r.h.s. being functional derivatives. With $\langle\mathbf{a}, \mathbf{b}\rangle$ and $\mathbf{a} \wedge \mathbf{b}$ designating the scalar and wedge products of the two vectors $\mathbf{a}$ and $\mathbf{b}$, with $\langle\boldsymbol{\nabla}$, for the divergence operator, this gives the continuity equation

$$
\partial_{t} \rho+\frac{1}{a^{2}}\langle\nabla, \rho \nabla S\rangle=0
$$

for the mass densities and, with the symbol $*$ designating the convolution product of two functions over $\mathbb{R}^{n}$, the non-local Bernoulli equation

$$
\partial_{t} S+\frac{1}{2 a^{2}}(\nabla S)^{2}+\frac{g}{a} \psi *\left(\rho-\rho_{b}\right)=0
$$

for the velocity potential. The velocity field being curl-free, Euler equation is

$$
\partial_{t} \mathbf{U}+\frac{1}{a^{2}}\langle\mathbf{U}, \boldsymbol{\nabla}\rangle \mathbf{U}+\frac{g}{a} \nabla \psi *\left(\rho-\rho_{b}\right)=0 .
$$

Furthermore, with $\Delta$, the Laplace operator and since $\Delta \psi=-\delta(\mathbf{x})$, it is useful to consider the so-called lap-Bernoulli or div-Euler equation which are local in the densities, namely

$$
\left\langle\boldsymbol{\nabla},\left(\partial_{t} \mathbf{U}+\frac{1}{a^{2}}\langle\mathbf{U}, \boldsymbol{\nabla}\rangle \mathbf{U}\right)\right\rangle-\frac{g}{a}\left(\rho-\rho_{b}\right)=0 .
$$

Notice here that, by introducing the peculiar velocity field $\mathbf{v}(\mathbf{x}, t)$ through $\mathbf{U}(\mathbf{x}, t)=$ $a(t) \mathbf{v}(\mathbf{x}, t)$ in $(2.5)$, the proper mass density $\mathcal{P}(\mathbf{x}, t)$ through $\rho(\mathbf{x}, t)=a(t)^{3} \mathcal{P}(\mathbf{x}, t)$ 
in (2.3) and (2.5) and putting $g=g_{a}(3)=-4 \pi G$ in (2.5), we obtain exactly the equations for single speed solutions in 3D of the Jeans-Vlasov-Poisson description of the Newtonian models, i.e. [3, eqn. 7] for example. If $f(\mathbf{x}, \mathbf{p}, t)$ is a Vlasovian distribution function on the 6-dimensional phase space $(\mathbf{x}, \mathbf{p})$, then its corresponding equation [3, eqn.3] admit single-speed solutions, [3, eqn. 6] in the form $f(\mathbf{x}, \mathbf{p}, t)=$ $a(t)^{3} \mathcal{P}(\mathbf{x}, t) \delta(\mathbf{p}-m a \mathbf{v}(\mathbf{x}, t))$.

For our purposes it is more convenient to introduce the particle velocity field $\mathbf{u}(\mathbf{x}, t)$ through $\mathbf{U}=a^{2} \mathbf{u}$. Then, (2.3) becomes

$$
\partial_{t} \rho+\langle\boldsymbol{\nabla}, \rho \mathbf{u}\rangle=0
$$

and, after division by $a^{2},(2.5)$ and (2.6) become, with $\dot{a}=d a / d t$,

$$
\begin{gathered}
\partial_{t} \mathbf{u}+2 \frac{\dot{a}}{a} \mathbf{u}+\langle\mathbf{u}, \boldsymbol{\nabla}\rangle \mathbf{u}+\frac{g}{a^{3}} \nabla \psi *\left(\rho-\rho_{b}\right)=0, \\
\left\langle\boldsymbol{\nabla},\left(\partial_{t} \mathbf{u}+2 \frac{\dot{a}}{a} \mathbf{u}+\langle\mathbf{u}, \boldsymbol{\nabla}\rangle \mathbf{u}\right)\right\rangle-\frac{g}{a^{3}}\left(\rho-\rho_{b}\right)=0 .
\end{gathered}
$$

For later comparison with the Lagrangian equation of motion (3.20), it is meaningfull to establish the PDE for $\partial_{t}^{2} \rho$. Introducing the mass current

$$
\mathbf{j}=\frac{\rho}{a^{2}} \boldsymbol{\nabla} S=\frac{\rho}{a^{2}} \mathbf{U}=\rho \mathbf{u},
$$

we have

$$
\partial_{t} \rho+\langle\nabla, \mathbf{j}\rangle=0
$$

and, in a form still containing $\nabla S$ via (2.10), to be later eliminated in function of generalized coordinates and their time derivatives,

$$
\partial_{t}^{2} \rho+2 \frac{\dot{a}}{a} \rho-\left\langle\boldsymbol{\nabla},\left(\langle\boldsymbol{\nabla}, \mathbf{j}\rangle \mathbf{u}+\langle\mathbf{j}, \boldsymbol{\nabla}\rangle \mathbf{u}+\frac{g}{a^{3}} \rho \boldsymbol{\nabla} \psi *\left(\rho-\rho_{b}\right)\right)\right\rangle=0 .
$$

\section{Lagrangian formulations of the Monge problem}

Interested in analytical tools aimed at dealing with different aspects of boundary value problems in time, in Fluid Dynamics, we present here four Lagrangian formulations of our models. The first one is directly connected to the Hamiltonian $H(S, \rho)$ of equation (2.2) in the sense that, by means of the inversion of the continuity equation (2.3) and of an appropriate Legendre transform, it leads to the 
Lagrangian $L_{1}\left(\partial_{t} \rho, \rho\right)$ given by (3.10). The second one is based on the idea to eliminate $-\nabla S$ instead of $S$ from the continuity equations in introducing vector valued applications $\boldsymbol{\eta}(\mathbf{x}, \mathbf{t})$ such that $\rho(\mathbf{x}, t)=\langle\boldsymbol{\nabla}, \boldsymbol{\eta}(\mathbf{x}, \mathbf{t})\rangle$. It leads to the Lagrangian $L_{2}\left(\partial_{t} \boldsymbol{\eta},\langle\boldsymbol{\nabla}, \boldsymbol{\eta}\rangle\right)$ given by (3.28). The third one is based on the introduction of an auxiliary vector valued application $\boldsymbol{\xi}(\mathbf{x}, t)$ that is an $n$-dimensional generalization of the Gelfand mass coordinate in $n=1$, in the sense that the $n$ components of $\boldsymbol{\xi}$ are constants of the motion like in [25, eqn. 1.11] or in [26, eqn. 3.4]. In this version, the mass density is given by the determinant of the Jacobian matrix $D_{\mathrm{x}} \boldsymbol{\xi}$, with elements $\left(D_{\mathbf{x}} \boldsymbol{\xi}\right)_{i, j}=\partial \boldsymbol{\xi}_{i} / \partial \mathbf{x}_{j}=\partial_{j} \boldsymbol{\xi}_{i}$ and the Lagrangian becomes $L_{3}\left(\partial_{t} \boldsymbol{\xi}\right.$, det $\left.\left(D_{\mathbf{x}} \boldsymbol{\xi}\right)\right)$ given by (3.36). The last version, most useful in practice, is based on the Lagrangian coordinates $\mathbf{x}(\boldsymbol{\xi}, t)$ of the characteristics of the models, parametrized by $\boldsymbol{\xi}$, and leads to the Lagrangian $L_{4}\left(\partial_{t} \mathbf{x}, \mathbf{x}\right)$ given by $(3.51)$.

\subsection{First version}

The aim is, here, to express $S$ as a function of $\rho$ and of $\partial_{t} \rho$. To do so, we start from the continuity equation (2.3) and re-write it in the form

$$
\partial_{t} \rho=\Omega S
$$

where $\Omega=-a^{-2}\langle\boldsymbol{\nabla}, \rho \boldsymbol{\nabla}\rangle$ is a Hermitian operator and a function of $\rho$ and $\boldsymbol{\nabla} \rho$. Next we introduce the symmetric kernel $K(\mathbf{x}, \mathbf{y}, \rho)$ in such a way that

$$
S(\mathbf{x})=\int d \mathbf{y} K(\mathbf{x}, \mathbf{y}, \rho) \partial_{t} \rho(\mathbf{y}):=K * \partial_{t} \rho .
$$

It follows that, formally: $\Omega \cdot K=\delta(\mathbf{x}-\mathbf{y})$, i.e., that

$$
-\frac{1}{a^{2}}\langle\boldsymbol{\nabla}, \rho(\mathbf{x}) \boldsymbol{\nabla} K(\mathbf{x}, \mathbf{y}, \rho)\rangle=\delta(\mathbf{x}-\mathbf{y}) .
$$

At this point, we introduce the $n$-dimensional kernel, generally non-symmetric,

$$
\mathbf{W}(\mathbf{x}, \mathbf{y}, \rho):=\frac{1}{a^{2}} \rho(\mathbf{x}) \nabla K(\mathbf{x}, \mathbf{y}, \rho)
$$

that satisfies the equation

$$
\langle\boldsymbol{\nabla}, \mathbf{W}(\mathbf{x}, \mathbf{y}, \rho)\rangle=-\delta(\mathbf{x}-\mathbf{y}),
$$

and we notice that

$$
\mathbf{j}=\mathbf{W} * \partial_{t} \rho
$$


The general solution of (3.5) for $n>1$ is established in the first Appendix. It is shown that $\mathbf{W}$ is a function of $\boldsymbol{\nabla} \ln \rho$ with the important consequence that its functional derivative with respect to $\rho$ vanishes. The explicit form of the solutions for $\mathbf{W}$ and $K$ in $1 \mathrm{D}$ are given below.

We consider next the kinetic part $T_{1}\left(\partial_{t} \rho, \rho\right)$ of the first Lagrangian. It is the Legendre transform of the kinetic part $T(\mathbf{S}, \rho)$ of the Hamiltonian (2.1). With $\rho(\boldsymbol{\nabla} S)^{2}=\langle\boldsymbol{\nabla},(S \rho \boldsymbol{\nabla} S)\rangle-S\langle\boldsymbol{\nabla}, \rho \boldsymbol{\nabla} S\rangle$, with the definition of $\Omega$ and in assuming, as usual, the vanishing of the surface integral of $S \rho \nabla S$, the kinetic part of the Hamiltonian (2.1) can also be written in the form

$$
T(S, \rho)=\frac{1}{2} \int d \mathbf{x} S(\mathbf{x}) \Omega(\rho(\mathbf{x})) S(\mathbf{x}) .
$$

Thus

$$
\begin{aligned}
T_{1}\left(\partial_{t} \rho, \rho\right) & =\sup _{S}\left(\int d \mathbf{x} \partial_{t} \rho(\mathbf{x}) S(\mathbf{x})-\frac{1}{2} \int d \mathbf{x} S(\mathbf{x}) \Omega(\rho(\mathbf{x})) S(\mathbf{x})\right) \\
& =\frac{1}{2} \int d \mathbf{x} \partial_{t} \rho(\mathbf{x}) S(\mathbf{x}),
\end{aligned}
$$

or

$$
T_{1}\left(\partial_{t} \rho, \rho\right)=\frac{1}{2} \int d \mathbf{x} d \mathbf{y} \partial_{t} \rho(\mathbf{x}) K(\mathbf{x}, \mathbf{y}, \rho) \partial_{t} \rho(\mathbf{y})
$$

and the generic Lagrangian associated to the first version is

$$
\begin{aligned}
L_{1}\left(\partial_{t} \rho, \rho\right)= & \frac{1}{2} \int d \mathbf{x} d \mathbf{y} \partial_{t} \rho(\mathbf{x}) K(\mathbf{x}, \mathbf{y}, \rho) \partial_{t} \rho(\mathbf{y}) \\
& -\frac{g}{2 a} \int d \mathbf{x} d \mathbf{y}\left(\rho(\mathbf{x})-\rho_{b}\right) \psi(|\mathbf{x}-\mathbf{y}|)\left(\rho(\mathbf{y})-\rho_{b}\right)
\end{aligned}
$$

Several investigations of theoretical interest can be based on (3.10) and (3.9). For example, setting $\rho=\rho_{b}$ in $K$ which becomes $\cong-a^{2}\left(\rho_{b} \Delta\right)^{-1} \delta(\mathbf{x}-\mathbf{y})=a^{2} \rho_{b}^{-1} \psi(|\mathbf{x}-\mathbf{y}|)$, or $\rho=\rho_{m}$ ( $m$ for mean value) if $\rho_{b}=0$ produces a quadratic approximation of $L_{1}$ in $\delta \rho(\mathbf{x})=\rho(\mathbf{x})-\rho_{b}$ or $\rho(\mathbf{x})-\rho_{m}$ that can be diagonalized by Fourier transform. With an interaction of positive type for instance, this gives an excellent approximation to the longitudinal collective excitations of the fluid [8, eqn. 45], and to the plasmons in the case of the OCP for example. Moreover, the Riemannian structure of formula (3.9) ought to be of interest in the mathematical analysis of the Monge problem of perfect fluids, as alluded to by Villani in [28, sect. 8.1.2].

It is thus appropriate to give some more details about $K(\mathbf{x}, \mathbf{y}, \rho)$. We notice first that, owing to the conservation of the total mass $M,(3.2)$ leaves us with the freedom 
of an additive constant in the kernel. We establish next a non-linear identity that $K$ has to satisfy. This identity results from the fact that the kinetic energy given by, (3.9) is linear in $K$ whereas that given in (2.1), with (3.2), gives a quadratic expression in $\nabla K$. Combining these two properties gives

$$
K(\mathbf{x}, \mathbf{y}, \rho)=\frac{1}{a^{2}} \int d \mathbf{z} \rho(\mathbf{z})\left(\left\langle\nabla_{\mathbf{z}} K(\mathbf{z}, \mathbf{x}, \rho), \nabla_{\mathbf{z}} K(\mathbf{z}, \mathbf{y}, \rho)\right\rangle-\left(\boldsymbol{\nabla}_{\mathbf{z}} K(\mathbf{z}, \mathbf{0}, \rho)\right)^{2}\right) .
$$

This is the fundamental integro-differential equation for $K$, with the property that $K(0,0, \rho)=0$. We have also that

$$
K(\mathbf{x}, \mathbf{y}, \rho)=a^{2} \int d \mathbf{z} \rho(\mathbf{z})^{-1}\left(\langle\mathbf{W}(\mathbf{z}, \mathbf{x}, \rho), \mathbf{W}(\mathbf{z}, \mathbf{y}, \rho)\rangle-\mathbf{W}(\mathbf{z}, \mathbf{0}, \rho)^{2}\right) .
$$

At this point, we can check that (3.11) satisfies (3.3).This is done in three steps. First, apply the operator $\Omega$ on the integrand of (3.11) and use (3.3). The term $\nabla_{\mathbf{z}}$ $\delta(\mathbf{z}-x)$ appears in the integrand. Next, integrate by part : there occur a vanishing surface integral and a remainder with a negative sign. Then, use again (3.3) and obtain a convolution of delta distributions, namely

$$
\Omega K=-\frac{1}{a^{2}}\langle\nabla, \rho \nabla\rangle K=\int d \mathbf{z} \delta(\mathbf{z}-\mathbf{x}) \delta(\mathbf{z}-\mathbf{y})=\delta(\mathbf{x}-\mathbf{y}) .
$$

As illustration, consider the 1D case. From (3.5) we have

$$
\mathbf{W}(\mathbf{x}, \mathbf{y})=-\frac{1}{2} \frac{\mathbf{x}-\mathbf{y}}{|\mathbf{x}-\mathbf{y}|}
$$

and from (3.4), we get

$$
K(\mathbf{x}, \mathbf{y}, \rho)=a^{2} \int_{\mathbf{y}}^{\mathbf{x}} d \mathbf{z} \rho(\mathbf{z})^{-1} \mathbf{W}(\mathbf{z}, \mathbf{y})=-a^{2} \frac{1}{2} \frac{(\mathbf{x}-\mathbf{y})}{|\mathbf{x}-\mathbf{y}|} \int_{\mathbf{y}}^{\mathbf{x}} d \mathbf{z} \rho(\mathbf{z})^{-1}
$$

It is easy to check that the same result is obtained from (3.12).

We proceed in establishing the Euler-Lagrange equation resulting from (3.10). It is

$$
\partial_{t} \frac{\delta L_{1}}{\delta \partial_{t} \rho}=\frac{\delta L_{1}}{\delta \rho}-\left\langle\nabla, \frac{\delta L_{1}}{\delta \nabla \rho}\right\rangle .
$$

Consider first the l.h.s of (3.16). We have

$$
\partial_{t} S=\left(\partial_{t} K\right) * \partial_{t} \rho+K * \partial_{t}^{2} \rho
$$


It is convenient to isolate the term $\partial_{t}^{2} \rho$ in applying the operator $\Omega$ on (3.17). Thus, and using the fact that $\Omega\left(\partial_{t} K\right)=-\left(\partial_{t} \Omega\right) K$, we get

$$
\Omega \partial_{t} S=\partial_{t}^{2} \rho-\left(\partial_{t} \Omega\right) K * \partial_{t} \rho=\partial_{t}^{2} \rho+2 \frac{\dot{a}}{a} \rho-\langle\nabla,\langle\nabla, \mathbf{j}\rangle \mathbf{u}\rangle
$$

with the help of (2.10), (2.11), (3.1) and (3.2).

Consider next the r.h.s. of (3.16) and use (3.12) for $K$, function of $\rho$ and $\mathbf{W}$. Since, as proved in the Appendix 5.1, W turns out to be a function of $\nabla \ln \rho$ with the property that the r.h.s. of (3.16) applied on a differentiable function of $\mathbf{W}(\mathbf{x}, \mathbf{y}, \boldsymbol{\nabla} \ln \rho)$ vanishes, the first term of this side only contributes to the EulerLagrange equation and, with (2.10) and (2.11) again, we find

$$
\frac{\delta L_{1}}{\delta \rho}=-\frac{1}{2} a^{2} \mathbf{u}^{2}-\frac{1}{a} \psi *\left(\rho-\rho_{b}\right) .
$$

Applying the operator $\Omega$ on (3.19) gives, for vorticity-free fluids, by dividing by $a^{2}$ and combining the result with (3.18), the final equation

$$
\partial_{t}^{2} \rho+2 \frac{\dot{a}}{a} \rho-\left\langle\boldsymbol{\nabla},\left(\langle\boldsymbol{\nabla}, \mathbf{j}\rangle \mathbf{u}+\langle\mathbf{j}, \boldsymbol{\nabla}\rangle \mathbf{u}+\frac{g}{a^{3}} \rho \boldsymbol{\nabla} \psi *\left(\rho-\rho_{b}\right)\right)\right\rangle=0 .
$$

which is exactly (2.12). However, notice that, here, we have $\mathbf{j}=\rho \mathbf{u}=\mathbf{W} * \partial_{t} \rho$.

It remains to focus on the Actions generally understood to be the generating functions of canonical transformations depending upon coordinates prescribed at initial and final times, $t_{0}$ and $t_{1}$, as time integral from $t_{0}$ to $t_{1}$ of Lagrangians depending upon the coordinates and their time derivatives and, also, as solutions of the ensuing Hamilton-Jacobi equations. In the present case, the coordinates are the densities with prescribed initial and final values $\left\{\rho_{0}\right\}$ and $\left\{\rho_{1}\right\}$; the Actions are symbolically written as $A_{1}(0,1):=A_{1}\left(\left\{\rho_{0}\right\}, t_{0} ;\left\{\rho_{1}\right\}, t_{1}\right)$ and the Hamilton-Jacobi equations are

$$
\partial_{t_{1}} A_{1}(0,1)+H\left(\frac{\delta A_{1}(0,1)}{\delta \rho_{1}} ; \rho_{1}\right)=0
$$

and

$$
-\partial_{t_{0}} A_{1}(0,1)+H\left(-\frac{\delta A_{1}(0,1)}{\delta \rho_{0}} ; \rho_{0}\right)=0 .
$$

Alternatively, with $T(S, \rho)$ given by (3.7), (3.21) becomes

$$
\begin{aligned}
& \partial_{t_{1}} A_{1}(0,1)+\frac{1}{2} \int d \mathbf{x} \frac{\delta A_{1}(0,1)}{\delta \rho_{1}(\mathbf{x})} \Omega\left(\rho_{1}(\mathbf{x})\right) \frac{\delta A_{1}(0,1)}{\delta \rho_{1}(\mathbf{x})} \\
& +\frac{g}{2} \int d \mathbf{x} d \mathbf{y}\left(\rho_{1}(\mathbf{x})-\rho_{b}\right) \psi(|\mathbf{x}-\mathbf{y}|)\left(\rho_{1}(\mathbf{y})-\rho_{b}\right)=0
\end{aligned}
$$


a similar equation being associated to (3.22).

At this point we should stress that Kantorovich [19], [20] has reformulated the Monge problem which, in the mean-time, had been studied by Appell [1], [2], as an optimal transport problem. Furthermore, for the case of interest here, (the so-called $L^{2}$ Kantorovich or Wasserstein distance) and on the basis of a seminal work on the polar factorization of certain vector fields by Brenier [5], [6], Benamou and Brenier [4] have proved, by means of an original fluid mechanical formulation, that the minimal cost of transportation equals the minimum of the Action $A_{1}(0,1)$ for a perfect fluid over the paths which lead from $\rho_{0}$ to $\rho_{1}$, paths which are solutions of $(3.20)$ with $g=0$ and $a=1$. An outstanding presentation of the results quoted above can be found in Villani's book [28, sects. 1, 2, subsects. 5.4.6, 8.1.1 \& 8.1.2] in particular.

\subsection{Second version}

Here, the idea is to eliminate $-\nabla S$ instead of $S$ from the continuity equation in introducing vector valued applications $\boldsymbol{\eta}(\mathbf{x}, \mathbf{t})$ such that

$$
\rho(\mathbf{x}, t)=\langle\boldsymbol{\nabla}, \boldsymbol{\eta}(\mathbf{x}, \mathbf{t})\rangle .
$$

With this Ansatz, the continuity equation (2.3) becomes

$$
\left\langle\boldsymbol{\nabla},\left(\partial_{t} \boldsymbol{\eta}+\frac{1}{a^{2}}\langle\boldsymbol{\nabla}, \boldsymbol{\eta}\rangle \boldsymbol{\nabla} S\right)\right\rangle=0 .
$$

Considering the homogenous solution of the expression in parenthesis, we get

$$
-\nabla S=a^{2} \frac{\partial_{t} \boldsymbol{\eta}}{\langle\boldsymbol{\nabla}, \boldsymbol{\eta}\rangle}
$$

and thus, the kinetic energy becomes

$$
T_{2}\left(\partial_{t} \boldsymbol{\eta},\langle\boldsymbol{\nabla}, \boldsymbol{\eta}\rangle\right)=\frac{1}{2} a^{2} \int d \mathbf{x} \frac{\left(\partial_{t} \boldsymbol{\eta}\right)^{2}}{\langle\boldsymbol{\nabla}, \boldsymbol{\eta}\rangle}
$$

In contrast with $T_{1}\left(\partial_{t} \rho, \rho\right)$ it is noticed that $T_{2}\left(\partial_{t} \boldsymbol{\eta},\langle\boldsymbol{\nabla}, \boldsymbol{\eta}\rangle\right)$ is local in the field variables and the corresponding Lagrangians read

$$
L_{2}\left(\partial_{t} \boldsymbol{\eta},\langle\boldsymbol{\nabla}, \boldsymbol{\eta}\rangle\right)=T_{2}\left(\partial_{t} \boldsymbol{\eta},\langle\boldsymbol{\nabla}, \boldsymbol{\eta}\rangle\right)-V\left(\langle\boldsymbol{\nabla}, \boldsymbol{\eta}\rangle-\rho_{b}\right) .
$$

At this point we observe that the vector field variable conjugated to $\boldsymbol{\eta}$, called $\boldsymbol{\pi}$, is

$$
\boldsymbol{\pi}=\boldsymbol{\delta} T_{2}\left(\partial_{t} \boldsymbol{\eta},\langle\nabla, \boldsymbol{\eta}\rangle\right) / \boldsymbol{\delta} \boldsymbol{\eta}=a^{2} \frac{\partial_{t} \boldsymbol{\eta}}{\langle\boldsymbol{\nabla}, \boldsymbol{\eta}\rangle},
$$


and we notice that

$$
\pi=-\nabla S
$$

It follows that the Hamiltonian associated to the pair $(\boldsymbol{\pi}, \boldsymbol{\eta})$ is

$$
H_{2}(\boldsymbol{\pi}, \boldsymbol{\eta})=\frac{1}{2 a^{2}} \int d \mathbf{x}\langle\boldsymbol{\nabla}, \boldsymbol{\eta}\rangle \boldsymbol{\pi}^{2}+V\left(\langle\boldsymbol{\nabla}, \boldsymbol{\eta}\rangle-\rho_{b}\right) .
$$

It is interesting to observe that Euler equations and the continuity equations in a divergence form can be put in a Hamiltonian form with $H_{2}(-\mathbf{U}, \boldsymbol{\eta})$ and that the final equation of motion (2.12) can also be put in a divergence form, with $\rho(\mathbf{x}, t)=$ $\langle\boldsymbol{\nabla}, \boldsymbol{\eta}(\mathbf{x}, \mathbf{t})\rangle, \mathbf{j}(\mathbf{x}, t)=-\partial_{t} \boldsymbol{\eta}(\mathbf{x}, \mathbf{t})$ and $\mathbf{u}(\mathbf{x}, t)=-\left(\partial_{t} \boldsymbol{\eta}(\mathbf{x}, \mathbf{t}) /\langle\boldsymbol{\nabla}, \boldsymbol{\eta}\rangle\right)$..

\subsection{Third version}

This version is based on the idea to generalize Gelfand mass coordinate in 1D [17, eqns $6.1 \& 6.9$, pp 318,319$]$ by the introduction of an $n$-dimensional vector valued application $\boldsymbol{\xi}(\mathbf{x}, t) \in C^{2}\left(\mathbb{R}^{n} \times \mathbb{R}, \mathbb{R}^{n}\right)$ such that each component is a constant of the motion,namely

$$
\partial_{t} \boldsymbol{\xi}+\langle\mathbf{u}, \boldsymbol{\nabla}\rangle \boldsymbol{\xi}=0
$$

where $\mathbf{u}(\mathbf{x}, t)$ is the particle velocity field occurring in (2.7). In fact,and despite their different physical meanings, these constants of the motion called here mass coordinates can be compared with the labeling coordinates introduced by Salmon [26, Section 2] in the sense that the mass densities are given by the same determinant [26, eqn. 2.2] and (3.33), and that both are relevant in the theory of irrotational fluids like those considered in this paper. Other sets of constants of the motion are the Clebsch potentials [15, p.2] and the constraints introduced by Lin [21, eqn. III.3.7], both relevant in the theory of rotational systems as emphasized in particular by Rylov [25] .

The crucial advantage of $\boldsymbol{\xi}(\mathbf{x}, t)$ is, indeed, that we can express the density $\rho(\mathbf{x}, t)$ as the determinant of the Jacobian matrix $D_{\mathbf{x}} \boldsymbol{\xi}$, i.e.,

$$
\rho(\mathbf{x}, t)=\operatorname{det}\left(D_{\mathbf{x}} \boldsymbol{\xi}(\mathbf{x}, t)\right),
$$

a relation known to satisfy the continuity equation (2.3) as recalled in the second Appendix for convenience. Notice also that if

$$
\boldsymbol{\xi}=\nabla \sigma
$$


then (3.33) is the Monge-Ampère equation and $\rho$ is the determinant of the Hessian matrix of $\sigma$. We proceed in expressing the velocity field $\mathbf{u}$ as a function of $\partial_{t} \boldsymbol{\xi}$ and $D_{\mathrm{x}} \boldsymbol{\xi}$. Noticing that $\langle\mathbf{u}, \boldsymbol{\nabla}\rangle \boldsymbol{\xi}=D_{\mathrm{x}} \boldsymbol{\xi} . \mathbf{u}$, we have

$$
\mathbf{u}=-\left(D_{\mathbf{x}} \boldsymbol{\xi}\right)^{-1} \partial_{t} \boldsymbol{\xi}
$$

The corresponding Lagrangian becomes

$$
\begin{array}{r}
L_{3}\left(\partial_{t} \boldsymbol{\xi}, \operatorname{det}\left(D_{\mathbf{x}} \boldsymbol{\xi}\right)\right)=\frac{a^{2}}{2} \int d \mathbf{x}\left(\operatorname{det}\left(D_{\mathbf{x}} \boldsymbol{\xi}\right)\right)\left(\left(D_{\mathbf{x}} \boldsymbol{\xi}\right)^{-1} \partial_{t} \boldsymbol{\xi}\right)^{2} \\
-\frac{1}{2 a} \iint d \mathbf{x} d \mathbf{y}\left(\left(\operatorname{det}\left(D_{\mathbf{x}} \boldsymbol{\xi}\right)\right)-\rho_{b}\right) \psi(|\mathbf{x}-\mathbf{y}|)\left(\left(\operatorname{det}\left(D_{\mathbf{x}} \boldsymbol{\xi}\right)\right)-\rho_{b}\right) .
\end{array}
$$

It is not necessary to derive again the equation of motion (2.12). It suffices to point out that, in the present version,

$$
\mathbf{j}=\rho \cdot \mathbf{u}=-\operatorname{det}\left(D_{\mathbf{x}} \boldsymbol{\xi}\right) \cdot\left(D_{\mathbf{x}} \boldsymbol{\xi}\right)^{-1} \partial_{t} \boldsymbol{\xi}
$$

However, it is instructive to illustrate solutions of the equation of motion for the simplest case labeled $\mathrm{PF}^{1}$. Considering one-dimensional problems at this point, we notice that,in fact, Kalman stream function $\psi[18$, section 2] is equal to -Gelfand mass coordinate! With $(\mathbf{x}, t) \in(\mathbb{R} \times \mathbb{R})$, with $\boldsymbol{\eta}(\mathbf{x}, t)=\boldsymbol{\xi}(\mathbf{x}, t)$ in the illustration, we have

$$
L_{3}\left(\partial_{t} \boldsymbol{\xi}, \partial_{x} \boldsymbol{\xi}\right)=\frac{1}{2} \int d \mathbf{x} \frac{\left(\partial_{t} \boldsymbol{\xi}\right)^{2}}{\partial_{\mathbf{x}} \boldsymbol{\xi}}
$$

and the equation of motion reads

$$
\partial_{t}^{2} \boldsymbol{\xi}=\partial_{\mathbf{x}}\left(\left(\partial_{t} \boldsymbol{\xi}\right)^{2} / \partial_{\mathbf{x}} \boldsymbol{\xi}\right)
$$

Notice here that if we set $\boldsymbol{\xi}=\partial_{\mathbf{x}} \sigma$, then the above equation becomes an homogenous Monge-Ampère equation for $\sigma$ in the variables $\boldsymbol{\xi}$ and $t$. We proceed by making the Ansatz

$$
\boldsymbol{\xi}(\mathbf{x}, t)=\boldsymbol{\xi}(\mathbf{z}(\mathbf{x}, t)) .
$$

It is easily shown that $\mathbf{z}(\mathbf{x}, t)$ satisfies exactly the same PDE than $\boldsymbol{\xi}$ i.e.

$$
\partial_{t}^{2} \mathbf{z}=\partial_{\mathbf{x}}\left(\left(\partial_{t} \mathbf{z}\right)^{2} / \partial_{\mathbf{x}} \mathbf{z}\right)
$$

An analysis of this PDE suggests that the sought solutions have to be a dimensionless ratio of a linear function of $\mathbf{x}$ and $t$ divided by a linear function of $t$. Setting $s=$ 
$\left(t-t_{0}\right) /\left(t_{1}-t_{0}\right), \mathbf{z}=\mathbf{z}(\mathbf{x}, s), \mathbf{z}(\mathbf{x}, 0)=\mathbf{x} / \alpha_{o}$ and $\mathbf{z}(\mathbf{x}, 1)=(\mathbf{x}-\mathbf{l}) / \alpha_{1}, \mathbf{l}$ being a shift defined below, we find

$$
\mathbf{z}(\mathbf{x}, s)=(\mathbf{x}-\mathbf{l} s) /\left(\alpha_{o}+\left(\alpha_{1}-\alpha_{o}\right) s\right) .
$$

It follows that

$$
\rho(\mathbf{x}, s)=\partial_{\mathbf{z}} \boldsymbol{\xi}(\mathbf{z}(\mathbf{x}, t)) /\left(\alpha_{o}+\left(\alpha_{1}-\alpha_{o}\right) s\right) .
$$

For example, if we choose $M=2$ and $\boldsymbol{\xi}(\mathbf{z})=\tanh (\mathbf{z})$, then

$$
\rho(\mathbf{x}, s)=\left(\alpha_{o}+\left(\alpha_{1}-\alpha_{o}\right) s\right)^{-1}\left(\cosh ^{2}(\mathbf{x}-\mathbf{l} s) /\left(\alpha_{o}+\left(\alpha_{1}-\alpha_{o}\right) s\right)^{-1}\right.
$$

with

$$
\begin{gathered}
\rho(\mathbf{x}, 0)=\left(\alpha_{o}\right)^{-1}\left(\cosh ^{2}(\mathbf{x}) /\left(\alpha_{o}\right)^{-1},\right. \\
\rho(\mathbf{x}, 1)=\left(\alpha_{1}\right)^{-1}\left(\cosh ^{2}(\mathbf{x}-\mathbf{l}) /\left(\left(\alpha_{1}\right)\right)^{-1}\right.
\end{gathered}
$$

and $\mathbf{l}$ is identified as the shift experienced by the barycenter of the mass distributions during the time interval $t_{1}-t_{o}$. For this example, the Lagrangian and the Action read, by setting $\tau=t_{1}-t_{0}$

$$
\begin{aligned}
L_{3}\left(\tau^{-1} \partial_{s} \boldsymbol{\xi}, \partial_{\mathbf{x}} \boldsymbol{\xi}\right) & =\frac{1}{2 \tau^{2}} \int d \mathbf{x} \frac{\left(\partial_{s} \boldsymbol{\xi}\right)^{2}}{\partial_{\mathbf{x}} \boldsymbol{\xi}} \\
& =\frac{1}{2 \tau^{2}} \frac{1}{\alpha_{o}+\left(\alpha_{1}-\alpha_{o}\right) s}\left(2 \mathbf{l}^{2}+\left(\alpha_{1}-\alpha_{o}\right)^{2} \frac{\pi^{2}}{6}\right)
\end{aligned}
$$

and

$$
\begin{aligned}
A_{2}(0,1) & =\tau \int_{0}^{1} d s L_{2}\left(\tau^{-1} \partial_{s} \boldsymbol{\xi}, \partial_{\mathbf{x}} \boldsymbol{\xi}\right) \\
& =\frac{1}{2 \tau}\left(2 \mathbf{l}^{2}+\left(\alpha_{1}-\alpha_{o}\right)^{2} \frac{\pi^{2}}{6}\right) \frac{1}{\alpha_{1}-\alpha_{0}} \ln \frac{\alpha_{1}}{\alpha_{0}} .
\end{aligned}
$$

Notice that if $\alpha_{1} \rightarrow \alpha_{0}$ and by restoring the fact that $2=M, A_{2}(0,1) \rightarrow \frac{1}{2} M 1^{2} / \tau$, which is the action of an undeformed mass distribution just translated during the time interval $\tau$ with the velocity $\mathbf{l} / \tau$.

An important remark has to be made here: it is that the Ansatz (3.40) characterizes solutions of the Monge problem that preserve the shape of the mass distributions during their transport, very much like the solutions of the Cauchy problem [9, p. 851 fig. 1], [10, p. 231, 234, 238, fig. 1, 3, 7], [12, p. 182 Ex. 5] in their domain of regularity. As of now and in the sequel all solutions of the models considered in this paper 
which preserve the shape of their mass distributions will be called monomorphic. By contrast, we will call dimorphic the solutions characterized by initial and final mass distributions being of different shape. More precisely, we consider one-parameter families of radial and normalized mass distributions $\sim \alpha^{-n} f_{n}(x / \alpha)$ which are such that, as $\alpha \rightarrow 0$, these distributions $\rightarrow \operatorname{Dirac} \delta^{n}(x)$ and we define by monomorphic the cases where both boundary densities belong to the same family and by dimorphic, the cases where the boundary densities belong to different families.By analogy with the concept of correlated and uncorrelated initial conditions utilized in previous work for solving the Cauchy problem,we could also speak here of correlated and uncorrelated boundary conditions Moreover, in order to insure the integrability of our examples and to have an easy access to their explicit and/or implicit solutions, only radially symmetric initial and final mass distributions will be considered as illustrated in the third Appendix This restriction defines the class of solutions announced in the title of this paper. Two subclasses: the monomorphic (correlated) and the dimorphic (uncorrelated) ones will be distinguished in the next section.

\subsection{Fourth version}

This is the most useful version when dealing with applications. It is in fact an outcome of the third version in the sense that the auxiliary vector valued application $\boldsymbol{\xi}$ will serve to parametrize the characteristics $\mathbf{x}(\boldsymbol{\xi}, t)$ of the different models. On the basis of (3.35) and from a known formula concerning partial derivatives, we have

$$
\mathbf{u}(\mathbf{x}, t)=-\left(D_{\mathbf{x}} \boldsymbol{\xi}\right)^{-1} \partial_{t} \boldsymbol{\xi}=\partial_{t} \mathbf{x}(\boldsymbol{\xi}, t)
$$

We have furthermore that

$$
\operatorname{det}\left(D_{\mathbf{x}} \boldsymbol{\xi}\right) d x=d \boldsymbol{\xi} \text {. }
$$

The new Lagrangian is consequently, with $\varpi(\boldsymbol{\xi})$ designating the range of $\boldsymbol{\xi}$,

$$
\begin{aligned}
L_{4}\left(\partial_{t} \mathbf{x}, \mathbf{x}\right)= & \frac{1}{2} a^{2} \int_{\varpi} d \boldsymbol{\xi}\left(\partial_{t} \mathbf{x}(\boldsymbol{\xi}, t)\right)^{2}-\frac{g}{2 a}\left(\int_{\varpi} d \boldsymbol{\xi} \int_{\varpi} d \boldsymbol{\xi}^{\prime} \psi\left(\left|\mathbf{x}(\boldsymbol{\xi})-\mathbf{y}\left(\boldsymbol{\xi}^{\prime}\right)\right|\right)\right. \\
& -2 \int_{\varpi} d \boldsymbol{\xi} \int d \mathbf{y} \psi(|\mathbf{x}(\boldsymbol{\xi})-\mathbf{y}|) \rho_{b} \\
& \left.+\iint d \mathbf{x} d \mathbf{y} \psi(|\mathbf{x}-\mathbf{y}|) \rho_{b}^{2}\right)
\end{aligned}
$$


The kinetic part of this Lagrangian with $a=1$ is precisely that of [26, eqn. 2.6].The equation of motion resulting from the above Lagrangian is, after division by $a^{2}$,

$\partial_{t}^{2} \mathbf{x}(\boldsymbol{\xi}, t)+2 \frac{\dot{a}}{a} \partial_{t} \mathbf{x}(\boldsymbol{\xi}, t)+\frac{g}{a^{3}}\left(\int_{\varpi} d \boldsymbol{\xi}^{\prime} \boldsymbol{\nabla} \psi\left(\left|\mathbf{x}(\boldsymbol{\xi})-\mathbf{y}\left(\boldsymbol{\xi}^{\prime}\right)\right|\right)-\int d \mathbf{y} \boldsymbol{\nabla} \psi(|\mathbf{x}(\boldsymbol{\xi})-\mathbf{y}|) \rho_{b}\right)=0$

Solutions of (3.52) are of the form $\mathbf{x}(\boldsymbol{\xi}, t)=\mathbf{x}\left(\mathbf{x}_{0}(\boldsymbol{\xi}), t_{0}, \mathbf{x}_{1}(\boldsymbol{\xi}), t_{1}, t\right)$ where $\mathbf{x}_{0}(\boldsymbol{\xi})$ and $\mathbf{x}_{1}(\boldsymbol{\xi})$ are the coordinates of the characteristics at $t=t_{0}$ and $t=t_{1}$. With these coordinates, we can determine the inverse of the mass densities $\widetilde{\rho}$ through

$$
\widetilde{\rho}(\boldsymbol{\xi}, t)^{-1}=\operatorname{det}\left(D_{\boldsymbol{\xi}} \mathbf{x}(\boldsymbol{\xi}, t)\right)
$$

and, similarly, for $\widetilde{\rho}_{0}(\boldsymbol{\xi})^{-1}$ and $\widetilde{\rho}_{1}(\boldsymbol{\xi})^{-1}$. On the other hand, we have $\rho(\mathbf{x}, t)=$ $\operatorname{det}\left(D_{\mathbf{x}} \boldsymbol{\xi}\right)$. Inversion of the function $\mathbf{x}(\boldsymbol{\xi}, t)$ into $\boldsymbol{\xi}(\mathbf{x}, t)$ is therefore necessary for determining

$$
\rho(\mathbf{x}, t)=\widetilde{\rho}(\boldsymbol{\xi}(\mathbf{x}, t), t) .
$$

In this version, the Action $A_{4}(0,1)$ reads :

$$
A_{4}(0,1)=A_{4}\left(\left\{\mathbf{x}_{0}\right\}, t_{0} ;\left\{\mathbf{x}_{1}\right\}, t_{1}\right)=\int_{t_{0}}^{t_{1}} d t L_{4}\left(\partial_{t} \mathbf{x}, \mathbf{x}\right)
$$

\section{Examples of integrable solutions of (3.52)}

Three properties are associated to the solutions of (3.52) considered in this section. The first one is general (for our class of Newtonian and Coulombian systems) in the sense that it is independent of the boundary mass densities chosen. It is that the motion of the barycenter of the mass distribution can be separated from that of its constituents relative to this center. This property follows from the linearity of the first two terms of (3.52), of the translational invariance of the third term of this equation and of the linearity also of its last term due to the fact that the potential due to the background term being quadratic in the coordinates of the test particle, the force field is linear in these coordinates. Designating by $\mathbf{b}(t)$ the coordinates of the barycenter, we set

$$
\mathbf{x}(\boldsymbol{\xi}, \mathbf{b}, t)=\mathbf{b}(t)+\mathbf{y}(\boldsymbol{\xi}, t),
$$

where $\mathbf{y}(\boldsymbol{\xi}, t)$ satisfies (3.52) with prescribed boundary conditions discussed below and where $\mathbf{b}(t)$ satisfies the generic equation

$$
\partial_{t}^{2} \mathbf{b}+2 \frac{\dot{a}}{a} \partial_{t} \mathbf{b}+\frac{g(n)}{a^{3} n} \rho_{b} \mathbf{b}=0
$$


where $\dot{a}:=d a / d t, \mathbf{b}\left(t_{0}\right)=0$ and $\mathbf{b}\left(t_{1}\right)=\mathbf{l}$.

The next two properties of our solutions follow from the assumption of spherically symmetric boundary densities $\rho_{i}(x), \widetilde{\rho}_{i}(\xi)$ with $i=0,1$. The first one is

$$
\mathbf{y}_{i}(\boldsymbol{\xi})=\frac{\boldsymbol{\xi}}{\xi} y_{i}(\xi)
$$

with non negative amplitudes $y_{i}(\xi)$.Equation (4.3) means that the initial and final coordinates are vectors parallel to $\boldsymbol{\xi}$. This does not mean that $\mathbf{y}(\boldsymbol{\xi}, t)$ be parallel to $\boldsymbol{\xi}$ for all times. In general and considering a reference system where $\boldsymbol{\xi}$ represents the $z$ axis, we can write $\mathbf{y}(\boldsymbol{\xi}, t)=\mathbf{y}_{\|}(\boldsymbol{\xi}, t)+\mathbf{y}_{\perp}(\boldsymbol{\xi}, t)$ and $\mathbf{y}_{\perp}(\boldsymbol{\xi}, t)$ has to satisfies $(3.52)$ with Dirichlet boundary conditions at $t=t_{0}$ and $t=t_{1}$. It is nevertheless clear that a non vanishing $\mathbf{y}_{\perp}(\boldsymbol{\xi}, t)$ will increase the kinetic part of the action associated to the corresponding trajectory and thus, according to Benamou and Brenier [4], that it will increase the "cost" of the mass transportation. In the spirit of the Monge problem, solutions with $\mathbf{y}_{\perp}(\boldsymbol{\xi}, t)=0$ should therefore be favored. Consequently, the assumption will be made in the sequel that $\mathbf{y}$ be parallel to $\boldsymbol{\xi}$ for all times.

The second one is that if $\mathbf{y}(\boldsymbol{\xi}, t)$ is a solution of (3.52) satisfying our boundary conditions, then $-\mathbf{y}(\boldsymbol{\xi}, t)$ is also a solution. Such pairs of symmetric trajectories that stem from the same set of initial conditions have been called sister trajectories in $[11$, pp. 178, 180] \& [13, p. 44]. The corresponding pairs of test particles with opposite initial coordinates and velocity may or may not collide. If they don't, their trajectories belong to the set of regular solutions. If they do at some collision time $t_{c}$ that may or may not be in the time interval $t_{1}-t_{0}=\tau$, the rules of the adhesion model $[10$, p.246] \& $[10$, ref.4, 6] tell us that they do not cross but merge, stick together and pursue their motion along the $\mathbf{y}=0$ or $\mathbf{x}=\mathbf{b}$ axis, generating a finite, a semiinfinite or even a periodic shock-line according to the force fields they are subject to, as illustrated in [10, fig. B.1.b] and [11, p. 178]. Needless to say, the mass densities are singular (Dirac measures) along the shock-line with time-dependent amplitude. Examples are given in [10, p, 236, 237, eqn. 28, fig. 6] and [12, p. 177 $\&$ 182] for solutions of the Cauchy problem in 1D. We can now proceed with the presentation of examples of solutions of the Monge problem which, as a consequence of our assumptions, will give rise to mass densities function of $|\mathbf{x}-\mathbf{b}|=|\mathbf{y}|$.

It is instructive to begin with the one-dimensional version of the models, in particular since their equations of motion are linear second order ODE's with or without time-dependant coefficient. A convenient way to establish their generic ODE is to start from $(2.9)$ in $1 \mathrm{D}$, to set $\rho=\partial_{\mathbf{x}} \boldsymbol{\xi}$, to integrate the resulting PDE, to set the constant of integration equal zero and lastly to go over to the equation of the char- 
acteristics. This procedure gives

$$
\partial_{t}^{2} \mathbf{x}(\boldsymbol{\xi}, t)+2 \frac{\dot{a}}{a} \partial_{t} \mathbf{x}(\boldsymbol{\xi}, t)-\frac{g(1)}{a^{3}}\left(\boldsymbol{\xi}-\rho_{b} \mathbf{x}(\boldsymbol{\xi}, t)\right)=0 .
$$

We proceed by considering the $P F^{1}, C_{1}^{1}$ and $N_{1}^{1}, C_{2}^{1}, N_{2}^{1}$ models, we present a short generalization concerning the $P F^{n}$ one since the corresponding ODE is also linear and leave aside the $N_{3}^{1}$ as well as the other models for $n>1$ one since they imply more elaborate numerical analysis.

\subsection{The $P F^{1}$ model}

Here: $a=1, g(1)=0, \partial_{t}^{2} \mathbf{b}=0$ and $\partial_{t}^{2} \mathbf{y}=0$. Setting $s=\left(t-t_{0}\right) /\left(t_{1}-t_{0}\right)$ gives

$$
\mathbf{b}(s)=s \mathbf{l}
$$

and

$$
\begin{aligned}
\mathbf{y}(\boldsymbol{\xi}, s) & =\frac{\boldsymbol{\xi}}{\xi}\left(y_{0}(\xi)(1-s)+y_{1}(\xi) s\right) \\
& :=\frac{\boldsymbol{\xi}}{\xi} y(\xi, s) .
\end{aligned}
$$

The regular set of solutions is characterized by $y(\xi, s)>0$, which means that pairs of sister trajectories do not collide. The singular set is characterized by the occurrence of shocklines $s(\xi)$, solution of $y(\xi, s)=0$,i.e.

$$
s(\xi)=\frac{y_{0}(\xi)}{y_{0}(\xi)-y_{1}(\xi)}=\left(1-\frac{y_{1}(\xi)}{y_{0}(\xi)}\right)^{-1} .
$$

With the examples given in the third Appendix, we may set

$$
y_{i}(\xi)=\alpha_{i} \zeta\left(\xi / \bar{\xi}, 1, k_{i}\right)
$$

where the amplitude $\alpha_{i}>0$ and we recall that the dimorphic cases are characterized by $k_{1} \neq k_{0}$ whereas $k_{0}=k_{1}$ correspond to the monomorphic ones, and (4.8) with (4.9) becomes

$$
s(\xi)=\left(1-\frac{\alpha_{1} \zeta\left(\xi / \bar{\xi}, 1, k_{1}\right)}{\alpha_{0} \zeta\left(\xi / \bar{\xi}, 1, k_{0}\right)}\right)^{-1} .
$$

At this point and in setting 


$$
\beta_{i}:=\left.\partial_{\xi} y_{i}(\xi)\right|_{\xi=0}=\left.\alpha_{i} \partial_{\xi} \zeta\left(\xi / \bar{\xi}, 1, k_{i}\right)\right|_{\xi=0},
$$

we notice that in the limit $\xi \rightarrow 0$

$$
s(0)=\left(1-\frac{\beta_{1}}{\beta_{0}}\right)^{-1} .
$$

This relation is true for all possible ratios of $\zeta\left(\xi / \bar{\xi}, 1, k_{i}\right)$. We show now that $(4.12)$ is also the value of the critical time $s_{c}$ which signals the onset of singular densities. We have indeed that, $\mathbf{b}(s)$ being independent of $\xi$,

$$
\widetilde{\rho}^{-1}(\xi)=\partial_{\xi}\left(y_{0}(\xi)(1-s)+y_{1}(\xi) s\right)
$$

and $\widetilde{\rho}^{-1}(\xi)=0$ implies that

$$
\begin{aligned}
s_{c} & =\left.\frac{\partial_{\xi} y_{0}(\xi)}{\partial_{\xi} y_{0}(\xi)-\partial_{\xi} y_{1}(\xi)}\right|_{\xi=0} \\
& =\lim _{\xi \rightarrow 0} \frac{y_{0}(\xi)}{y_{0}(\xi)-y_{1}(\xi)} \\
& =\frac{\beta_{0}}{\beta_{0}-\beta_{1}}=s(0)
\end{aligned}
$$

according to L'Hospital rule. This result means that, at criticality, the monomorphic and dimorphic cases coincide. The last step is to determine the mass density $\rho(x, s)=\widetilde{\rho}(\xi(x, s), s)$. This implies to solve for $\xi(y, s)$ the equation

$$
\begin{aligned}
y & =\left(y_{0}(\xi)(1-s)+y_{1}(\xi) s\right) \\
& =\alpha_{0} \zeta\left(\xi / \bar{\xi}, 1, k_{0}\right)(1-s)+\alpha_{1} \zeta\left(\xi / \bar{\xi}, 1, k_{1}\right) s .
\end{aligned}
$$

In the dimorphic cases the solutions of this implicit equation requires numerical analysis. Fig.4.1 shows an illustration for the density profile corresponding to $\beta_{0}=1$, $k_{0}=1, \beta_{1}=2, k_{1}=2$ and $l=6$ In this case, it turns out that an explicit solution can be given for $s=1 / 2$, that the critical time $s_{c}=-1$ and that the regular solutions ceases to exist for $s \leq-1$. Symmetrically if we had chosen $k_{0}=2, \beta_{0}=2$ and $k_{1}=1, \beta_{1}=1$, the regular solutions would cease to exist in the future after $s \geq 2$. In the monomorphic case we have

$$
|\mathbf{x}-s \mathbf{l}|=y=\alpha(s) \zeta(\xi / \bar{\xi}, 1, k)
$$




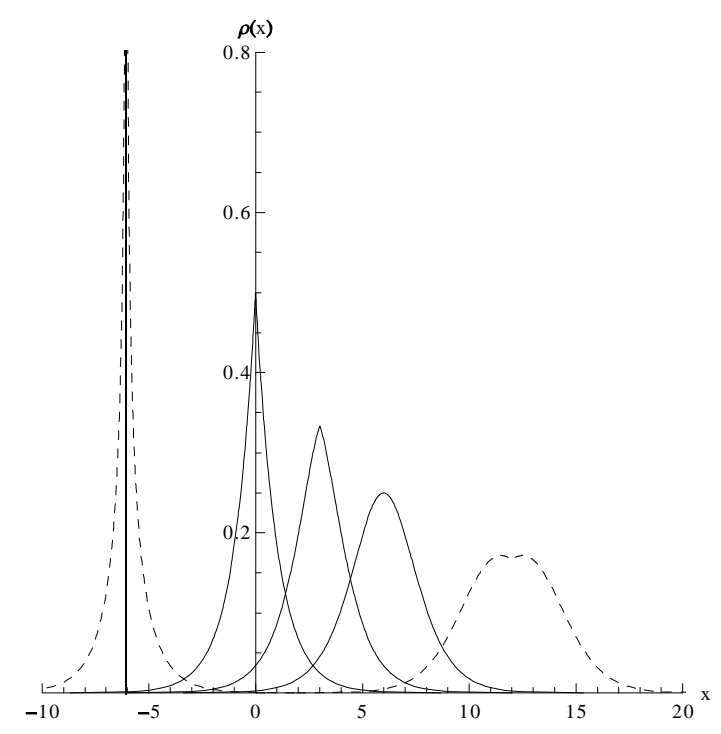

Figure 4.1: $\rho(x)$ at different times for the $P F^{1}$ model

where $\alpha(s)=a_{0}(1-s)+\alpha_{1} s$, and thus, for $k=2$ for instance

$$
\rho(x, 1,2)=\frac{M}{2} \frac{1}{\alpha(s)} \frac{1}{\cosh ^{2}(|\mathbf{x}-s \mathbf{l}| / \alpha(s))}
$$

in agreement with (3.44) for $M=2$. It is clear that as $s \rightarrow-1, \rho(x, 1,2) \rightarrow$ $M \delta(\mathbf{x}+\mathbf{l})$.

\subsection{The $C_{1}^{1}$ and $N_{1}^{1}$ models}

In this case: $a=1, g(1)=\left\{g_{r}(1),-g_{a}(1)\right\}, \rho_{b}=0, \partial_{t}^{2} \mathbf{b}=0$ and $\partial_{t}^{2} y=g \xi$. The solutions are, in setting again $s=\left(t-t_{0}\right) /\left(t_{1}-t_{0}\right)$ and $\gamma=\frac{1}{2} g(1)\left(t_{1}-t_{0}\right)^{2}, \mathbf{b}=s \mathbf{l}$ for the barycenter and

$$
y(\xi, s)=y_{0}(\xi)(1-s)+y_{1}(\xi) s-\xi \gamma s(1-s)
$$

for the coordinates relative to the barycenter. Here again the regular set is charac-

terized by $y>0$ and for singular solutions the shockline $s(\xi)$ are solutions of the quadratic equation

$$
y_{0}(\xi)(1-s(\xi))+y_{1}(\xi) s(\xi)-\xi \gamma s(\xi)(1-s(\xi))=0 .
$$


As $\xi \rightarrow 0$ we obtain for $s_{c}$, after division by $\gamma \neq 0$, the equation

$$
s_{c}^{2}-\left(1-\frac{\beta_{1}-\beta_{0}}{\gamma}\right) s_{c}+\frac{\beta_{0}}{\gamma}=0 .
$$

The solutions of the above equation are

$$
s_{c}^{ \pm}=\frac{1}{2}\left(1-\frac{\beta_{1}-\beta_{0}}{\gamma}\right) \pm \sqrt{\Delta}
$$

with the discriminant

$$
\begin{aligned}
\Delta & =\frac{1}{4}\left(1-\frac{\beta_{1}-\beta_{0}}{\gamma}\right)^{2}-\frac{\beta_{0}}{\gamma} \\
& =\frac{1}{4}\left(1-2 \frac{\left(\beta_{1}+\beta_{0}\right)}{\gamma}+\frac{\left(\beta_{1}-\beta_{0}\right)^{2}}{\gamma^{2}}\right) \\
& =\frac{1}{4}\left(1-\frac{\gamma^{-}}{\gamma}\right)\left(1-\frac{\gamma^{+}}{\gamma}\right),
\end{aligned}
$$

with

$$
\gamma^{ \pm}=\left(\sqrt{\beta_{1}} \pm \sqrt{\beta_{0}}\right)^{2}
$$

It follows that the solutions of (4.18) are regular as long as $\gamma \in\left(\gamma^{-}, \gamma^{+}\right)$and we have

$$
s_{c}^{ \pm}=\frac{1}{2}\left(1-\frac{\beta_{1}-\beta_{0}}{\gamma}\right) \pm \frac{1}{2}\left(1-2 \frac{\left(\beta_{1}+\beta_{0}\right)}{\gamma}+\frac{\left(\beta_{1}-\beta_{0}\right)^{2}}{\gamma^{2}}\right)^{1 / 2} .
$$

We have also that $s_{c}^{-}=\frac{\beta_{0}}{\beta_{0}-\beta_{1}}+o(\gamma)$ and $s_{c}^{+}=\frac{\beta_{0}-\beta_{1}}{\gamma}-\frac{\beta_{0}}{\beta_{0}-\beta_{1}}+o(\gamma)$, and thus as $\gamma \rightarrow 0, s_{c}^{+} \rightarrow \infty$.

We have noticed that it is only in the repulsive cases $\left(C_{1}^{1}\right)$ and for $\gamma \in\left(\gamma^{-}\right.$, $\gamma^{+}$) that regular solutions exist for all times. In all other cases there are pairs of critical times $s_{c}^{ \pm}(\gamma)$ from which, as time proceeds, either singular solutions emerge and disappear or, conversely, disappear and re-emerge.The corresponding density distributions, normalized to the total mass $M$, consist of a Dirac peak and of density tails with time-dependant amplitudes, that of the Dirac peak $\rightarrow M$ as $s \rightarrow \pm \infty$ in the attractive case $\left(N_{1}^{1}\right)$, but remaining $<M$ and lying in the interior of the time interval $(0,1)$ in the repulsive case $\left(C_{1}^{1}\right)$.

It is also interesting to consider, at criticality, the case with $\beta_{0}=\beta_{1}$ that can be set $=1$ without loss of generality. In this case, (4.20) becomes simply, for $\gamma \neq 0$

$$
s_{c}^{2}-s_{c}+1 / \gamma=0
$$




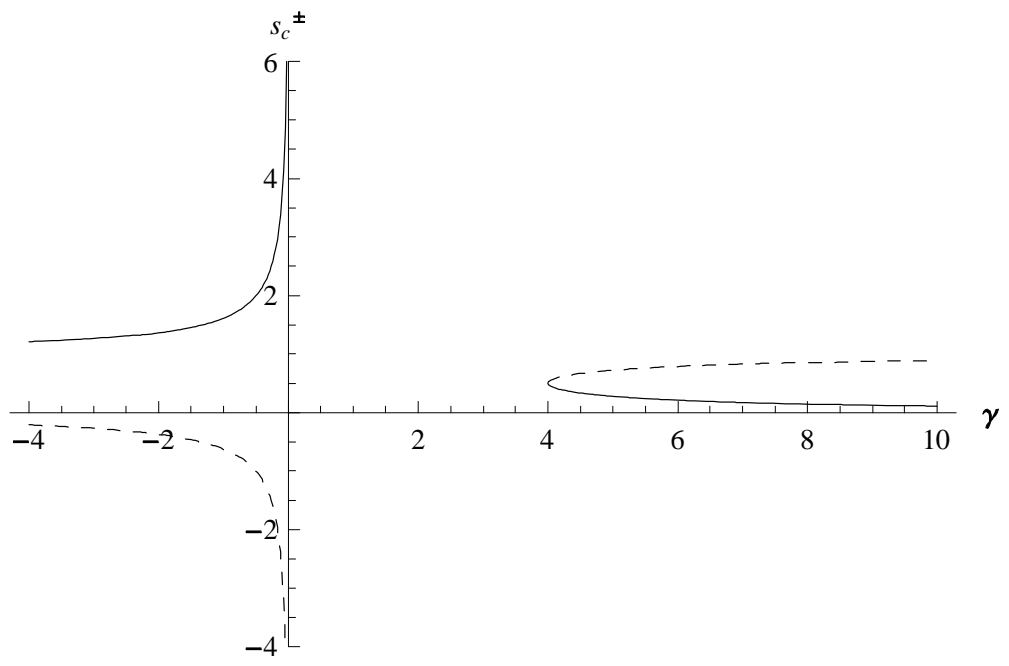

Figure 4.2: Regularity interval and singularity domains for $\beta_{0}=\beta_{1}=1$.

i.e.

$$
s_{c}^{ \pm}=1 / 2\left(1 \pm(1-4 / \gamma)^{1 / 2}\right)
$$

Fig.4.2 shows the graph of $s_{c}^{ \pm}(\gamma)$ given by (4.26). It is noticed that whatever the choice of the basis functions $\zeta(1, \xi, k)$, the solutions $s_{c}^{+}$and $s_{c}^{-}$lie in the interval $(0,1)$ for $\gamma \geq 4$ and outside this interval for $\gamma<0$.

It is finally worth considering the monomorphic case with $k_{0}=k_{1}=2$ and $\beta_{0}=\beta_{1}$ for example. Indeed, restoring the vectorial character of the variables in $1 \mathrm{D}$ , setting $\theta(s)=-\gamma s(1-s), \bar{\xi}=1$, i.e. $M=2$, we have

$$
\begin{aligned}
\mathbf{x}-\mathbf{l} \mathbf{s}= & \mathbf{y}=\quad \theta(s) \boldsymbol{\xi}+(\boldsymbol{\xi} / 2 \xi) \ln ((1+\xi) /(1-\xi)) \\
= & \theta(s) \boldsymbol{\xi}+\mathbf{1} / 2 \ln ((1+\boldsymbol{\xi}) /(1-\boldsymbol{\xi}))
\end{aligned}
$$

or

$$
\boldsymbol{\xi}=\tanh (\mathbf{y}-\theta(s) \boldsymbol{\xi})
$$

in complete analogy with [10, eqns. 26 and 24]. In fact (4.28) holds also for the velocity field $\mathbf{v}(\mathbf{x}, \theta)$ that satisfies the Burgers type of equation [10, eqn. 19] and solves a Cauchy problem with $\mathbf{v}(\mathbf{x}, 0)$ chosen so that $\rho(\mathbf{x}, 0) \sim \partial_{\mathbf{x}} \mathbf{v}(\mathbf{x}, 0)$, the said correlated initial conditions that played an important role in previous work $[10, \mathrm{p}$. 230, 232, 236], [11, section 1 footnote p. 173] and [13, p. 42]. Notice also that 
$\rho(\mathbf{x}, \theta)=\partial_{\mathbf{x}} \boldsymbol{\xi}(\mathbf{x}, \theta) \sim \partial_{\mathbf{x}} \mathbf{v}(\mathbf{x}, \theta)$ which means that for Burgers type of equations $\boldsymbol{\xi}$ $\sim \mathbf{v}$ up to a proportionality constant, and we recall here the quantitative analysis and illustrations given in [9, p. 851 fig. 1, 2], in [10, p. 237, 246 fig. 6, B.1] as well as the exact expressions for the density profiles of singular solutions given in $[9, \mathrm{p}$. 851 eqn. 42], in [10, p. 236, eqn. 28] and in [12, p. 177].

\subsection{The $C_{2}^{1}$ model}

Here, $a=1, g(1)=g_{r}(1), \rho_{b}>0, \rho_{b} g_{r}=\Omega_{P}^{2}$ and the corresponding equations are

$$
\partial_{t}^{2} \mathbf{b}+\Omega_{P}^{2} \mathbf{b}=\mathbf{0}
$$

and

$$
\partial_{t}^{2} \mathbf{y}+\Omega_{P}^{2}\left(\mathbf{y}-\rho_{b}^{-1} \boldsymbol{\xi}\right)=0 .
$$

Setting $\varphi=\Omega_{p} t=\Omega_{p}\left(t_{0}+\left(t_{1}-t_{0}\right) s\right)=\varphi_{0}+\phi s$, where $\phi=\varphi_{1}-\varphi_{0}=\left(\rho_{b} g_{r}\right)^{1 / 2}\left(t_{1}-t_{0}\right)$ is a coupling constant playing here the same role as $\gamma^{1 / 2}$ in the $C_{1}^{1}$ model, the relevant solutions of the above equations are

$$
\mathbf{b}(\phi, s)=\frac{\sin (\phi s)}{\sin \phi} \mathbf{l}
$$

and, setting $\mathbf{y}=(\boldsymbol{\xi} / \xi) y$,

$$
y(\xi, s)=\rho_{b}^{-1} \xi+(\sin \phi)^{-1}\left(( y _ { 0 } ( \xi ) - \rho _ { b } ^ { - 1 } \xi ) \operatorname { s i n } \left(\phi(1-s)+\left(y_{1}(\xi)-\rho_{b}^{-1} \xi\right) \sin (\phi s)\right.\right.
$$

We have also,b being independent of $\xi$,

$\widetilde{\rho}(\xi, s)^{-1}=\rho_{b}^{-1}+(\sin \phi)^{-1}\left(\left(\widetilde{\rho}_{0}(\xi)^{-1}-\rho_{b}^{-1}\right) \sin (\phi(1-s))+\left(\widetilde{\rho_{1}}(\xi)^{-1}-\rho_{b}^{-1}\right) \sin (\phi s)\right)$

The equilibrium properties of this model being $\rho_{b}^{-1}$ periodic, it is natural to consider also $\rho_{b}^{-1}$ periodic boundary densities. Measuring the latter in unit of $\rho_{b}$, one possibility is to periodize the density given in the first example of the third appendix. Shifting for convenience the origin of the $\mathrm{x}$ axis by half a period, we have

$$
\rho(x)=\sum_{n \in Z} \frac{M}{2 \alpha} \exp (-(1 / \alpha)|\mathbf{x}-\mathbf{n}-1 / 2|)=\frac{M}{2 \alpha} \frac{C h(x / \alpha)}{S h(1 / 2 \alpha)}, 0<x<1 / 2
$$


We have next

$$
\mu(x)=2 \int_{0}^{x} d x^{\prime} \rho\left(x^{\prime}\right)=2 \xi(x)=M S h(x / \alpha) / S h(1 / 2 \alpha)=M \xi / \bar{\xi}
$$

with $M=2 \xi(1 / 2)=2 \bar{\xi}$. It follows that

$$
x / \alpha=\ln \left(\xi S h(1 / 2 \alpha) / \bar{\xi}+\left(\left(1+(\xi S h(1 / 2 \alpha) / \bar{\xi})^{2}\right)^{1 / 2}\right):=\zeta(\xi S h(1 / 2 \alpha) / \bar{\xi}, 1,4) .\right.
$$

Another case to be considered is a density with compact support and $\rho_{b}^{-1}$ periodic. One example is

$$
\rho(x)=\frac{2 M}{\alpha}\left(1-\frac{2 x}{\alpha}\right), x \leq \alpha / 2,0<\alpha \leq 1
$$

and

$$
\rho(x)=0, \alpha / 2<x \leq 1 / 2
$$

Then,with $\bar{\xi}=M / 2$,

$$
\mu(x) / M=\xi / \bar{\xi}=\frac{4}{\alpha} x\left(1-\frac{x}{\alpha}\right)
$$

and

$$
\frac{x}{\alpha}=\frac{1}{2}\left(1-(1-\xi / \bar{\xi})^{1 / 2}\right):=\zeta(\xi / \bar{\xi}, 1,5) .
$$

With $\zeta_{i}(\xi / \bar{\xi})$ designating one of the basis functions described above, the general solution for this model is

$$
\left.y(\xi, s)=\xi+(\sin \phi)^{-1}\left(\left(\alpha_{0} \zeta_{0}(\xi / \bar{\xi})-\xi\right) \sin (\phi(1-s))+\left(\alpha_{1} \zeta_{1}(\xi / \bar{\xi})-\xi\right)\right) \sin (\phi s)\right)
$$

and the equation for $s_{c}$ is, with $\phi \neq 0, \beta_{0}>0, \beta_{1}>0$,

$$
\widetilde{\rho}\left(0, s_{c}\right)^{-1}=1+(\sin \phi)^{-1}\left(\left(\beta_{0}-1\right) \sin \left(\phi\left(1-s_{c}\right)+\left(\beta_{1}-1\right) \sin \left(\phi s_{c}\right)\right)=0 .\right.
$$

As illustration consider the situation along the diagonal $\beta_{0}=\beta_{1}=\beta$.In this case, we have

$$
\widetilde{\rho}\left(0, s_{c}\right)^{-1}=1-(\cos (\phi / 2))^{-1}\left((1-\beta) \cos \left(\phi\left(s_{c}-1 / 2\right)\right)=0\right.
$$

or

$$
2 s_{c}-1=\frac{2}{\phi} \arccos \left(\frac{1}{1-\beta} \cos \left(\frac{\phi}{2}\right)\right) .
$$

It is clear that singular solutions exist in the domain defined by $\left|\frac{1}{1-\beta} \cos \left(\frac{\phi}{2}\right)\right| \leq 1$.In the $(\beta, \phi)$ plane it is given by $\beta \leq 1-\left|\cos \frac{\phi}{2}\right|$ for $0<\beta \leq 1$ and by $\beta \geqslant 1+\left|\cos \frac{\phi}{2}\right|$ for $\beta \geqslant 1$. 


\subsection{The $N_{2}^{1}$ model.}

Here, $a(t)=\left(t / \tilde{t}_{0}\right)^{2 / 3}, g(1)=-g_{a}(1), \rho_{b}>0, g_{a} \rho_{b}=\Omega_{J}^{2}=\frac{2}{3} \tilde{t}_{0}^{-2}$. Noticing that $\frac{g_{a} \rho_{b}}{a(t)^{3}}=\frac{2}{3} t^{-2}$, the equations of motion become

$$
\partial_{t}^{2} \mathbf{b}+\frac{4}{3 t} \partial_{t} \mathbf{b}-\frac{2}{3 t^{2}} \mathbf{b}=\mathbf{0}
$$

and

$$
\partial_{t}^{2} \mathbf{y}+\frac{4}{3 t} \partial_{t} \mathbf{y}-\frac{2}{3 t^{2}}\left(\mathbf{y}-\frac{\boldsymbol{\xi}}{\rho_{b}}\right)=\mathbf{0}
$$

The Ansatz $\sim t^{p}$ gives $p(p-1)+\frac{4}{3} p-\frac{2}{3}=0$, i.e. $p=\frac{2}{3}$ and $p=-1$, the so-called growing and decaying modes, respectively. The corresponding eigenfunctions, $f_{0}(t)$ and $f_{1}(t)$ are such that $f_{0}\left(t_{0}\right)=1, f_{0}\left(t_{1}\right)=0$ and $f_{1}\left(t_{0}\right)=0, f_{1}\left(t_{1}\right)=1$. One finds

$$
f_{0}(t)=\frac{t_{0}\left(t_{1}^{5 / 3}-t^{5 / 3}\right)}{t\left(t_{1}^{5 / 3}-t_{0}^{5 / 3}\right)}
$$

and

$$
f_{1}(t)=\frac{t_{1}\left(t^{5 / 3}-t_{0}^{5 / 3}\right)}{t\left(t_{1}^{5 / 3}-t_{0}^{5 / 3}\right)}
$$

The solutions of the equations of motion become accordingly

$$
\mathbf{b}(t)=f_{0}(t) \mathbf{l}
$$

and, setting again $\mathbf{y}=(\boldsymbol{\xi} / \xi) y$,

$$
y(\xi, t)=\rho_{b}^{-1} \xi+\left(y_{o}(\xi)-\rho_{b}^{-1} \xi\right) f_{0}(t)+\left(y_{1}(\xi)-\rho_{b}^{-1} \xi\right) f_{1}(t)
$$

We have also

$$
\widetilde{\rho}(\xi, t)^{-1}=\partial_{\xi} y(\xi, t)=\rho_{b}^{-1}+\left(\widetilde{\rho_{0}}(\xi)^{-1}-\rho_{b}^{-1}\right) f_{0}(t)+\left(\widetilde{\rho_{1}}(\xi)^{-1}-\rho_{b}^{-1}\right) f_{1}(t)
$$

For the boundary densities, we can choose local deviations from $\rho_{b}$ with vanishing mean values and require that $\xi(x)$ be invertible. A two parameter family of such densities is, f.i.,given by:

$$
\rho(x)=\rho_{b}\left(1+\kappa\left(1-\left(\frac{x}{\alpha}\right)^{q}\right) \exp \left(-\frac{\left(\frac{x}{\alpha}\right)^{q}}{q}\right)\right), q>0,0<\kappa<\frac{\exp \frac{1+q}{q}}{q} .
$$


The corresponding $\xi(x)$ is:

$$
\xi(x)=\int_{0}^{x} d x \prime \rho(x \prime)=\rho_{b} x\left(1+\kappa \exp \left(-\frac{\left(\frac{x}{\alpha}\right)^{q}}{q}\right)\right)
$$

and

$$
\frac{x}{\alpha}:=\zeta\left(\frac{\xi}{\alpha \rho_{b}}, \kappa, q\right)
$$

With $\zeta_{i}\left(\frac{\xi}{\alpha \rho_{b}}\right)=\zeta\left(\frac{\xi}{\alpha \rho_{b}}, \kappa_{i}, q_{i}\right)$ designating any one of the basis functions defined above and with $y_{i}=\alpha_{i} \zeta_{i}$, the solution for $y(\xi, t)$ becomes

$$
y(\xi, t)=\rho_{b}^{-1} \xi+\left(\alpha_{0} \zeta_{0}\left(\frac{\xi}{\alpha \rho_{b}}\right)-\rho_{b}^{-1} \xi\right) f_{0}(t)+\left(\alpha_{1} \zeta_{1}\left(\frac{\xi}{\alpha \rho_{b}}\right)-\rho_{b}^{-1} \xi\right) f_{1}(t)
$$

and the equation for the critical times $t_{c}$ is

$$
\rho_{b} \widetilde{\rho}\left(0, t_{c}\right)^{-1}=1+\left(\beta_{0}-1\right) f_{0}\left(t_{c}\right)+\left(\beta_{1}-1\right) f_{1}\left(t_{c}\right)=0,
$$

or, invoking $f_{0}(t)$ and $f_{1}(t)$,

$$
t_{c}\left(t_{1}^{5 / 3}-t_{0}^{5 / 3}\right)+\left(\beta_{0}-1\right) t_{0}\left(t_{1}^{5 / 3}-t_{c}^{5 / 3}\right)+\left(\beta_{1}-1\right) t_{1}\left(t_{c}^{5 / 3}-t_{0}^{5 / 3}\right)=0
$$

A complete analysis of this equation lies outside the scope of this paper. As illustration,we consider the diagonal situation $\beta_{0}=\beta_{1}=\beta$.This gives,after re-arrangement of the terms,

$$
\left(t_{1}^{5 / 3}-t_{0}^{5 / 3}\right) t_{c}+(\beta-1)\left(\left(t_{1}-t_{0}\right) t_{c}^{5 / 3}+t_{0} t_{1}\left(t_{1}^{2 / 3}-t_{o}^{2 / 3}\right)\right)=0 .
$$

Introducing the dimension-less parameter $\vartheta_{1}=\frac{t_{1}}{t_{o}}>1$ and the variables $\vartheta=\frac{t}{t_{0}}, \vartheta_{c}=$ $\frac{t_{c}}{t_{0}}>0$, the above equation becomes

$$
\left(\vartheta_{1}^{5 / 3}-1\right) \vartheta_{c}+(\beta-1)\left(\left(\vartheta_{1}-1\right) \vartheta_{c}^{5 / 3}+\vartheta_{1}\left(\vartheta_{1}^{2 / 3}-1\right)\right)=0
$$

It is clear that for $\beta>1$ there are no real solution of $\vartheta_{c}$, all solutions are threrefore regular. It is also interesting to observe that for the un-physical value of $\beta=0$, we have the two solutions: $\vartheta_{c}=1$ and $\vartheta_{c}=\vartheta_{1}$. They act as separatrices. The two branches of real solutions for $\beta=0.1,0.3$, and 0.5 , shown in the Fig.4.3,are then easily understood.For a given $\vartheta_{1}$, we have two roots: $\vartheta_{c}^{-}<1$ and $\vartheta_{c}^{+}>\vartheta_{1}$. The singular set of solutions for $\widetilde{\rho}(\xi(x, t), t)=\rho(x, t)$ is precisely defined by $\vartheta \leq \vartheta_{c}^{-}$and $\vartheta \geqslant \vartheta_{c}^{+}$ whereas the regular set of solutions is defined by $\vartheta_{c}^{-}<\vartheta<\vartheta_{c}^{+}$. This interesting situation is reminiscent of the behavior noticed in the self-gravitating $\left(N_{1}^{1}\right)$ model in which singular solutions occur outside the time interval $\left(t_{0}, t_{1}\right)$. 


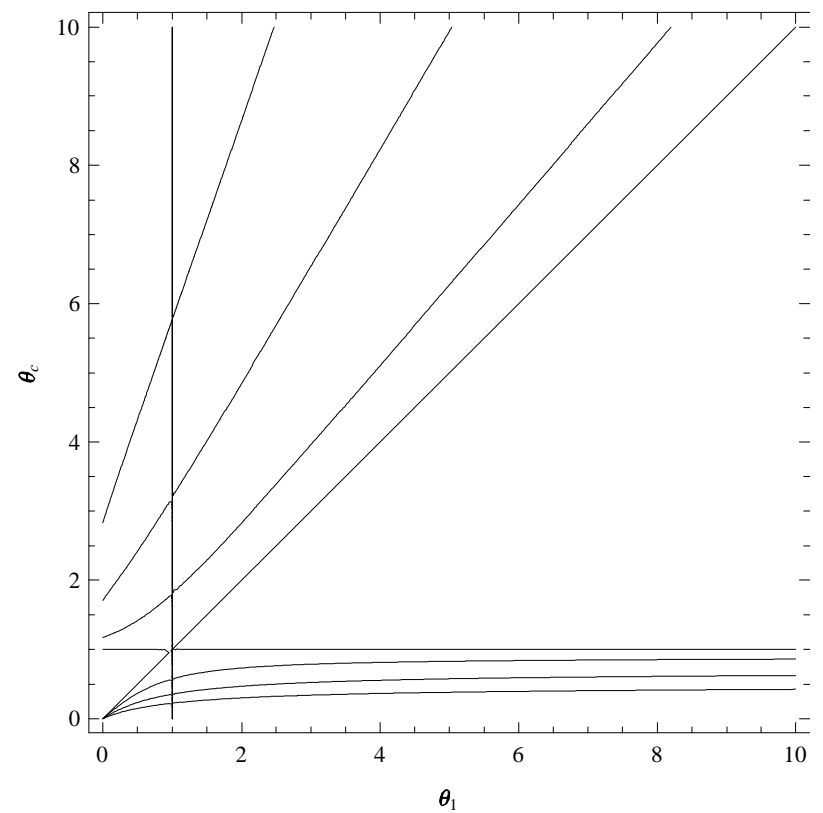

Figure 4.3: The critical time $\theta_{c}$ as a function of $\theta_{1}$ for 4 values of the parameter $\beta$.

\subsection{The $P F^{n}$ model for $n>1$}

In these cases we have again

$$
\mathbf{b}(s)=s \mathbf{l}
$$

and

$$
\mathbf{y}(\boldsymbol{\xi}, s)=\frac{\boldsymbol{\xi}}{\xi}\left(y_{0}(\xi)(1-s)+y_{1}(\xi) s\right):=\frac{\boldsymbol{\xi}}{\xi} y(\xi, s) .
$$

where $y_{i}(\xi)=\alpha_{i} \zeta\left(\xi / \bar{\xi}, n, k_{i}\right)$ given in the third Appendix. For $\widetilde{\rho}(\xi, n)^{-1}$ we have next and this is a general property for the class of solutions of the Monge problem dealt with in this paper,

$$
\widetilde{\rho}(\xi, s, n)^{-1}=\frac{1}{n \xi^{n-1}} \partial_{\xi} y(\xi, s)^{n}=\frac{1}{\xi^{n-1}} y(\xi, s)^{n-1} \partial_{\xi} y(\xi, s)
$$

and we notice that the equation for $s_{c}$ is, like in $1 \mathrm{D}$, given by $y(0, s)=0$. For the present model,we have

$$
\widetilde{\rho}\left(0, s_{c}, n\right)^{-1}=\left(\beta_{0}\left(1-s_{c}\right)+\beta_{1} s_{c}\right)^{n}=0
$$


with $\beta_{i}=\alpha_{i} \bar{\xi}^{-1}$ for the three examples given in the third Appendix. It is also from the equations of the characteristics relative to the barycenter, namely $y=y(\xi, s)$, which are invertible, that $\xi=\xi(y, s)$ has to be evaluated for constructing the densities $\widetilde{\rho}(\xi(y, s), s, n)=\rho(y, s)$ and then to insert $y=|\mathbf{x}-\mathbf{l} s|$. As illustration consider the monomorphic case for $k=2$. We have

$$
\rho(\mathbf{x}, s, n, 2)=\left(\bar{\xi} /\left(\alpha_{0}(1-s)+\alpha_{1} s\right)\right)^{n}\left(\cosh \left(|\mathbf{x}-\mathbf{l} s| /\left(\alpha_{0}(1-s)+\alpha_{1} s\right)\right)^{n}\right)^{-2} .
$$

In the dimorphic case,numerical analysis is required. For the case dealt with under $P F^{1}$, we find, for $n=2$ and setting $\bar{\xi}=1$ for simplicity, i.e. $\alpha_{0}=\beta_{0}=1, \alpha_{1}=$ $\beta_{1}=2$ and also $k_{0}=1, k_{1}=2$, the results shown in the figures 4.4 and 4.5 , namely $\rho\left(x_{1}, x_{2}=0, s\right)$, with $\mathbf{x}=\left(x_{1}, x_{2}\right), \rho\left(x_{1}=l s, x_{2}, s\right)$. For $n>2$ the behavior of the corresponding density profiles are qualitatively similar [22, section 3$]$.

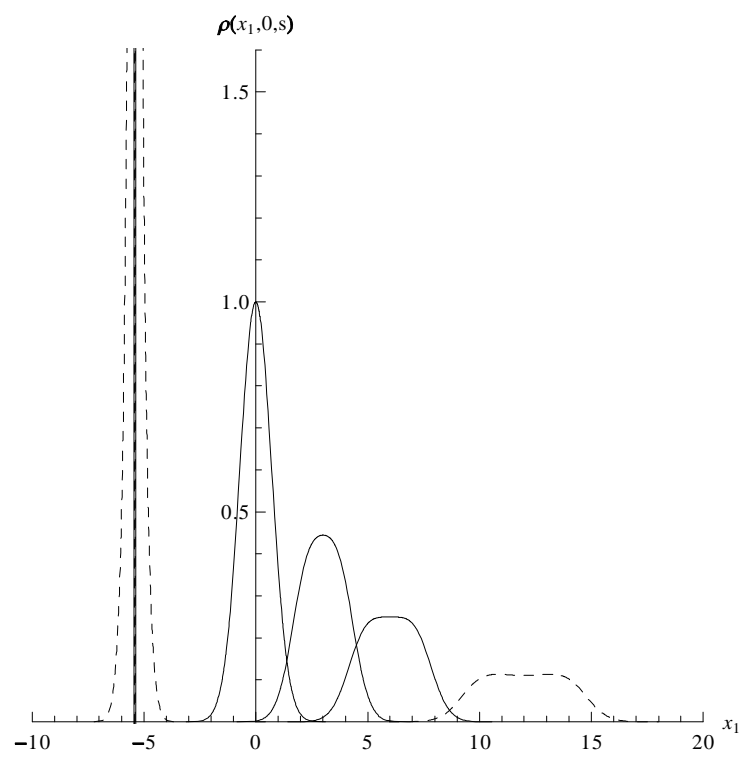

Figure 4.4: $\rho\left(x_{1}, 0, s\right)$ for the $P F^{2}$ model for $s=-0.9,0,0.5,1,2$.

So far, we have illustrated only solutions of linear ODE's of our models It is clear that many more integrable solutions of non linear ODE's can be envisaged. In addition,we see open problems, also computational,with more general boundary densities like those known in reconstruction problems in cosmology for instance, as suggested in [22, sect. 3] ; with systems with vorticity, with dissipative systems and, as conclusion, we suggest as exercise to solve the problem posed by Monge at the 


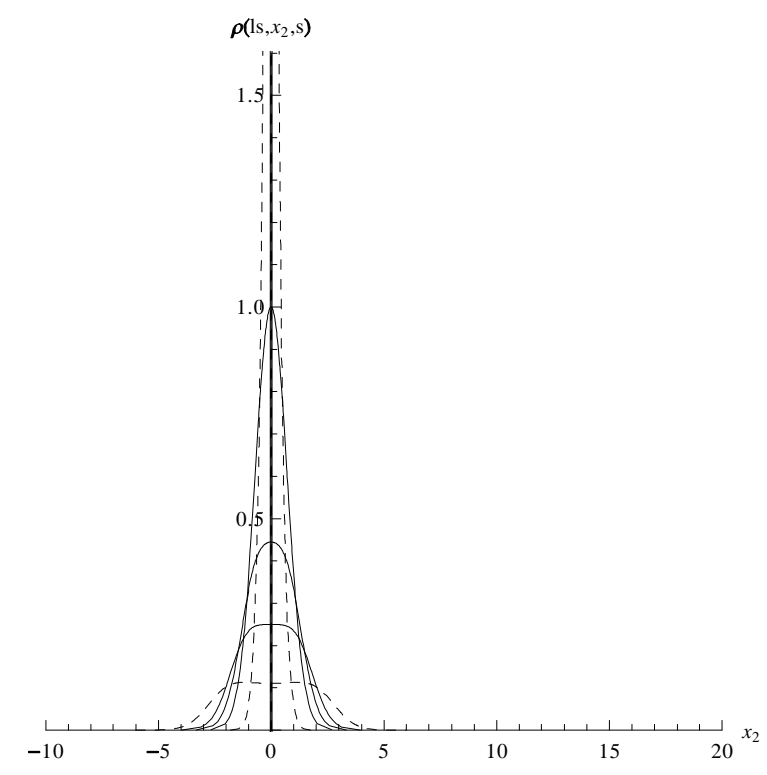

Figure 4.5: $\rho\left(l s, x_{2}, s\right)$ for the $P F^{2}$ model for $s=-0.9,0,0.5,1,2$

end of his Mémoire [23, p704], that is to take into account the effect of gravity in the transport of masses!

Acknowledgements: We are very much indebted to J. Stubbe for his lucid and sustained interest in our work and in particular, for his essential contribution to the elaboration of the third version of the Lagrangian formulations. The invaluable help provided by $M$. Vuffray for his fruitful collaboration in the presentation of the manuscript, in the numerical analysis of the equations and in the graphical representations figuring in the text is dutifully acknowledged. We wish also to thank heartily C. Villani for stimulating discussions held in 2009, in Prague and in Luminy concerning, in particular, the barycenter theorem and the first version of the Lagrangians, proposed to Y. Brenier, G. Loeper and A. Sobolevskii in 2006 at EPFL.

\section{Appendices}

Three appendices quoted in the main text are presented below. 


\subsection{PDE for $\mathbf{W}(\mathbf{x}, \mathbf{y}, \rho)$ with $1<n \leq 3$}

We start from (3.4) and (3.5), i.e.,

$$
\mathbf{W}(\mathbf{x}, \mathbf{y}, \rho)=\frac{1}{a^{2}} \rho(\mathbf{x}) \nabla K(\mathbf{x}, \mathbf{y}, \rho)
$$

and

$$
\langle\boldsymbol{\nabla}, \mathbf{W}(\mathbf{x}, \mathbf{y}, \rho)\rangle=-\delta(|\mathbf{x}-\mathbf{y}|)=\Delta \psi(|\mathbf{x}-\mathbf{y}|) .
$$

We make the Ansatz

$$
\mathbf{W}(\mathbf{x}, \mathbf{y}, \rho)=\nabla \psi(|\mathbf{x}-\mathbf{y}|)+\nabla \wedge \tilde{\mathbf{A}}(\mathbf{x}, \mathbf{y}, \rho) .
$$

Thus, and since $\langle\boldsymbol{\nabla}, \tilde{\mathbf{A}}(\mathbf{x}, \mathbf{y}, \rho)\rangle$ can be set to vanish,

$$
\nabla \wedge \mathbf{W}(\mathbf{x}, \mathbf{y}, \rho)=-\Delta \tilde{\mathbf{A}}(\mathbf{x}, \mathbf{y}, \rho)
$$

On the other hand,

$$
\boldsymbol{\nabla} \wedge \mathbf{W}(\mathbf{x}, \mathbf{y}, \rho)=\frac{1}{a^{2}}\langle\nabla \rho \wedge \nabla K(\mathbf{x}, \mathbf{y}, \rho)\rangle=\langle\nabla \ln \rho \wedge \mathbf{W}(\mathbf{x}, \mathbf{y}, \rho)\rangle,
$$

and, using the Ansatz for $\mathbf{W}(\mathbf{x}, \mathbf{y}, \rho)$ and introducing the vector field

$$
\chi(\rho):=\nabla \ln \rho=\frac{\nabla \rho}{\rho},
$$

we get

$$
-\Delta \tilde{\mathbf{A}}(\mathbf{x}, \mathbf{y}, \rho)-\chi(\rho) \wedge \nabla \wedge \tilde{\mathbf{A}}(\mathbf{x}, \mathbf{y}, \rho)=\chi(\rho) \wedge \nabla \psi|\mathbf{x}-\mathbf{y}| .
$$

It follows that $\mathbf{W}(\mathbf{x}, \mathbf{y}, \rho)$ and $\tilde{\mathbf{A}}(\mathbf{x}, \mathbf{y}, \rho)$ are functions of $\boldsymbol{\chi}(\rho)$ and thus, that the r.h.s. of (3.16) applied on any differentiable function of $\mathbf{W}$ or $\tilde{\mathbf{A}}$, vanishes, as claimed in section 3 , since

$$
\left(\frac{\partial}{\partial \rho}-\sum_{i=1}^{n} \partial_{j} \frac{\partial}{\partial \partial_{j} \rho}\right)\left(\frac{\partial_{i} \rho}{\rho}\right)=0, i=1, \ldots, n
$$




\subsection{Continuity equation for $\rho(\mathrm{x}, t)$}

We start from (3.33),i.e.

$$
\rho(\mathbf{x}, t)=\operatorname{det}\left(\partial_{1} \boldsymbol{\xi}, . . \partial_{j} \boldsymbol{\xi}, \ldots \partial_{n} \boldsymbol{\xi}\right) .
$$

Using the equations (3.32) for $\xi$,we compute

$$
\begin{aligned}
\partial_{t} \rho(\mathbf{x}, t) & =\sum_{j=1}^{n} \operatorname{det}\left(\partial_{1} \boldsymbol{\xi}, . . \partial_{j} \partial_{t} \boldsymbol{\xi}, \ldots \partial_{n} \boldsymbol{\xi}\right) \\
& =-\sum_{j, k=1}^{n} \operatorname{det}\left(\partial_{1} \boldsymbol{\xi}, . . \partial_{j} u_{k} \partial_{k} \boldsymbol{\xi}, \ldots \partial_{n} \boldsymbol{\xi}\right) \\
& =-\sum_{k=1}^{n}\left(u_{k} \partial_{k}+\left(\partial_{k} u_{k}\right)\right) \rho(\mathbf{x}, t)-\sum_{j \neq k=1}^{n}\left(\partial_{j} u_{k}\right) \operatorname{det}\left(\partial_{1} \boldsymbol{\xi}, . . \partial_{k} \boldsymbol{\xi}, \ldots \partial_{n} \boldsymbol{\xi}\right) \\
& =-\sum_{k=1}^{n} \partial_{k}\left(u_{k} \rho(\mathbf{x}, t)\right)+0
\end{aligned}
$$

i.e., the continuity equation (2.7), the remaining determinants vanishing since two of their columns are identical.

\subsection{Explicit $\xi$ of $\mathrm{x}$ relations for radially symmetric boundary densities}

Let $|\mathbf{x}|=x,|\boldsymbol{\xi}|=\xi$ be the radial coordinate of any spherically symmetric boundary mass densities in $n \mathrm{D}: \rho(x, n)$ and $\widetilde{\rho}(\xi, n)$ that satisfy the invertibility relations

$$
\rho(x, n)=\widetilde{\rho}(\xi(x), n)
$$

and

$$
\widetilde{\rho}(\xi, n)=\rho(x(\xi), n) .
$$

We set

$$
\boldsymbol{\xi}(\mathbf{x})=\nabla \sigma(x, n)=\frac{\mathbf{x}}{x} \partial_{x} \sigma(x, n)=: \mathbf{x} \phi(x, n)
$$

and thus, $\boldsymbol{\xi}$ being parallel to $\mathbf{x}$,

$$
\xi(x)=x \phi(x, n) .
$$


We need next

$$
\left(D_{\mathbf{x}} \boldsymbol{\xi}(\mathbf{x})\right)_{i j}=\delta_{i j} \phi(x, n)+x_{i} x_{j} \frac{1}{x} \partial_{x} \phi(x, n)
$$

and thus

$$
\begin{aligned}
\rho(x, n) & =\operatorname{det}\left(D_{\mathbf{x}} \boldsymbol{\xi}(\mathbf{x})\right) \\
& =\phi(x, n)^{n}+\frac{\langle\mathbf{x}, \mathbf{x}\rangle}{x} \phi(x, n)^{n-1} \partial_{x} \phi(x, n),
\end{aligned}
$$

all determinants with elements $\propto x_{i} x_{j}$ and of order $\geq 2$ vanishing since the $i^{\text {th }}$ line and column $(i \in\{1, . ., n\})$ are identical. Thus

$$
\begin{aligned}
\rho(x, n) & =\phi(x, n)^{n}+x \phi(x, n)^{n-1} \partial_{x} \phi(x, n) \\
& =\frac{1}{n x^{n-1}} \partial_{x}\left(x^{n} \phi(x, n)^{n}\right)
\end{aligned}
$$

or with (5.14)

$$
\rho(x, n)=\frac{1}{n x^{n-1}} \partial_{x} \xi(x)^{n} .
$$

It is useful to identify the above equation as the radial divergence of $\xi(x)^{n}$.Let us introduce here the mass coordinate $\mu(x, n)$. If $\omega_{n}$ designates the surface of an $n$ dimensional unit sphere, we have

$$
\begin{aligned}
\mu(x, n) & =\omega_{n} \int_{0}^{x} d x^{\prime} x^{\prime n-1} \rho\left(x^{\prime}, n\right) \\
& =\frac{\omega_{n}}{n} \xi(x)^{n} .
\end{aligned}
$$

and, if $\mu(\infty, n)=M$, we get for the range of $\xi$

$$
\xi \leq\left(\frac{M n}{\omega_{n}}\right)^{\frac{1}{n}}:=\bar{\xi},
$$

so that

$$
\mu(x, n)=M(\xi / \bar{\xi})^{n} .
$$

Similarly we set

$$
\mathbf{x}(\boldsymbol{\xi})=\boldsymbol{\nabla} \widetilde{\sigma}(\xi, n)=\frac{\boldsymbol{\xi}}{\xi} \partial_{\xi} \widetilde{\sigma}(\xi, n)=: \boldsymbol{\xi} \widetilde{\phi}(\xi, n)
$$


i.e.,

$$
x(\xi)=\xi \widetilde{\phi}(\xi, n) .
$$

Then and by analogy with the previous case

$$
\begin{aligned}
\widetilde{\rho}(\xi, n)^{-1} & =\operatorname{det}\left(D_{\boldsymbol{\xi}} \mathbf{x}(\boldsymbol{\xi})\right) \\
& =\frac{1}{n \xi^{n-1}} \partial_{\xi} x(\xi)^{n}
\end{aligned}
$$

and

$$
\widetilde{\mu}(\xi, n)^{-1}=\frac{\omega_{n}}{n} x^{n}(\xi) .
$$

We proceed by giving three examples of explicit $\boldsymbol{\xi}$ of $\mathbf{x}$ relations and of their corresponding boundary densities $\rho(x, n, k)$ and $\widetilde{\rho}(\xi, n, k), k \in\{1,2,3\}$.

\subsubsection{Example 1}

$$
\begin{aligned}
\rho(x, n, 1) & =(\bar{\xi} / \alpha)^{n} e^{-(x / \alpha)^{n}} \\
\mu(x, n, 1) & =M\left(1-e^{-(x / \alpha)^{n}}\right) \\
& =M(\xi / \bar{\xi})^{n} .
\end{aligned}
$$

Therefore

$$
\xi(x, 1)^{n}=\bar{\xi}^{n}\left(1-e^{-(x / \alpha)^{n}}\right)
$$

and thus

$$
(x / \alpha)^{n}=-\log \left(1-(\xi / \bar{\xi})^{n}\right):=\zeta(\xi / \bar{\xi}, n, 1)^{n} .
$$

We have also

$$
\widetilde{\rho}(\xi, n, 1)^{-1}=(\alpha / \bar{\xi})^{n}\left(1-(\xi / \bar{\xi})^{n}\right)^{-1} .
$$

It is easy to check that

$$
\rho(x(\xi), n, 1)=\widetilde{\rho}(\xi, n, 1) .
$$

\subsubsection{Example 2}

$$
\begin{aligned}
\rho(x, n, 2)=(\bar{\xi} / \alpha)^{n}\left(\cosh (x / \alpha)^{n}\right)^{-2} \\
\begin{aligned}
\mu(x, n, 2) & =M \tanh (x / \alpha)^{n} \\
& =M(\xi / \bar{\xi})^{n}
\end{aligned}
\end{aligned}
$$


thus

$$
\begin{gathered}
(x / \alpha)^{n}=\frac{1}{2} \log \left(\frac{1+(\xi / \bar{\xi})^{n}}{1-(\xi / \bar{\xi})^{n}}\right):=\zeta(\xi / \bar{\xi}, n, 2)^{n} \\
\widetilde{\rho}(\xi, n, 2)^{-1}=(\alpha / \bar{\xi})^{n} \frac{1}{1-(\xi / \bar{\xi})^{2 n}} .
\end{gathered}
$$

\subsubsection{Example 3}

$$
\begin{gathered}
\rho(x, n, 3)=(\bar{\xi} / \alpha)^{n} \frac{1}{\left(1+\left(\frac{x}{\alpha}\right)^{n}\right)^{2}} \\
\mu(x, n, 3)=M \frac{(x / \alpha)^{n}}{1+(x / \alpha)^{n}} \\
=M(\xi / \bar{\xi})^{n} \\
(x / \alpha)^{n}=\frac{(\xi / \bar{\xi})^{n}}{1-(\xi / \bar{\xi})^{n}}:=\zeta(\xi / \bar{\xi}, n, 3)^{n} \\
\widetilde{\rho}(\xi, n, 3)^{-1}=(\alpha / \bar{\xi})^{n} \frac{1}{\left(1-(\xi / \bar{\xi})^{n}\right)^{2}} .
\end{gathered}
$$

\section{References}

[1] P. Appell, Mémoire sur les déblais et les remblais des systèmes continus ou discontinus, Académie des Sciences de l'Institut de France, Paris, 29 (1887), 1-208.

[2] — Le problème géométrique des déblais et des remblais. Mémorial des Sciences Mathématiques, Académie des sciences de Paris, Fasc.27, (1928), 6-39. Available like the preceeding reference online at gallica.bnf.fr.

[3] E.Aurell, D. Fanelli, S. N. Gurbatov and A. Yu. Moshkov. Physica D 146,(2003) ,171-184.

[4] J. D. Benamou, Y. Brenier, A computational fluid mechanics solution to the Monge-Kantorovich mass transfer problem. Num. Math. 84, (2000), 375-393. 
[5] Y. Brenier, Décomposition polaire et réarrangement monotone des champs de vecteurs. C. R. Acad. Sci. Paris, t. 305, Série I, (1987), 805-808.

[6] - Polar factorization and monotone rearrangement of vector valued functions. Comm. Pure Appl. Math. 44, 4, 305, (1991) 375-417.

[7] — U. Frisch, M. Hennon, G. Loeper, S. Matarrese, R. Mohayaee and A. Sobolevskii, Reconstruction of the early Universe as a convex optimization problem,Mon. Not. R. Astron. Soc. 346, (2003), 501-524.

[8] Ph. Choquard, Toward a canonical-Hamiltonian and microscopic field theory of classical liquids,. Physica A 279 (2000) 45-59.

[9] - J. Wagner, On the «Mean-Field» interpretation of Burgers'equation, J.Stat.Phys. 116, Nos1/4, (2004) 843-853.

$[10]-, \longrightarrow$, On a class of implicit solutions of the continuity and Euler's equations for 1D systems with long range interactions I, Physica D 201 (2005) $230-248$.

$[11] \longrightarrow,-$ On a class of implicit solutions of the continuity and Euler's equations for 1D systems with long range interactions II, Physica D 226 (2007) 173-184.

[12] - T. Strömberg, A one-dimensional inviscid and compressible fluid in a harmonic potential well, Acta Appl Math 99 (2007), 161-183.

[13] - Single-speed solutions of the Vlasov-Poisson equations for Coulombian and Newtonian systems in $n \mathrm{D}$, Communications in Nonlinear Science and Numerical Simulation 13 (2008) 40-45.

[14] A. Clebsch, Ueber eine allgemeine Transformation der hydrodynamischen Gleichungen. Journal Fuer die reine und angewandte Mathematik, Vol. 54, (1857), 293-312.

[15] - Ueber die Integration der hydrodynamischen Gleichungen. Journal fuer die reine und angewandte Mathematik, Vol. 56, (1859),1-10.

[16] P. Coles, The State of the Universe, Nature 433, (2005), 248-255.

[17] I.M. Gelfand, Some problems in the theory of quasilinear equations, AMS Transl. (2), 29, (1963), 295-386; original paper in Uspekhi Mat. Nauk 14:2 (1959), 87158. 
[18] G. Kalman, Oscillations and Nonstationary Flow in a Zero Temperature Plasma, Annals of Physics, 10,(1960),1-10.

[19] L. V. Kantorovich, On the translocation of masses. J. Math. Sci. 133, No 4,(2006),1381-1382; original paper in Dokl. Akad. Nauk. SSSR No 7-8, (1942), 227-229.

[20] - On a problem of Monge. J. Math. Sci. 133, No 4,(2006), 1383; original paper in Uspekhi Mat. Nauk, 3,No 2, (1948), 225-226.

[21] C. C. Lin.Hydrodynamics of Helium II.Proc.Int.Sch Phys. Course XXI, ( Liquid Helium, Varenna), Academic, New York, (1963), 93-146

[22] R. Mohayaee, A. Sobolevskii, The Monge-Ampère-Kantorovich approach to reconstruction in cosmology, Physica D 237 (2008) 2145-2150.

[23] G. Monge, Mémoire sur la théorie des déblais et des remblais. Histoire de l'Académie Royale des Sciences de Paris, (1781), 666-704

[24] P. J. E. Peebles. The Large-Scale Structure of the Universe. Princeton University press (1980).

[25] Y.A. Rylov, Hydrodynamic equations for incompressible inviscid fluids in terms of generalized stream function , IJMMS, Vol 2004 (2004), No 11,541-570.

[26] R. Salmon, Hamiltonian fluid mechanics,Ann.Rev.Fluid Mech.20 (1988),225256.

[27] R. Taton, L'oeuvre scientifique de Monge. 7. Le problème des déblais et des remblais, .Presses Universitaires de France, (1951), 193-204.

[28] C. Villani, Topics in Optimal Transportation. Graduate Studies in Mathematics, AMS. Vol 58 (2003). 